\title{
The genome-wide impact of cadmium on microRNA and mRNA expression in contrasting $\mathrm{Cd}$ responsive wheat genotypes
}

Min Zhou ${ }^{1,2,3}$, Shigang Zheng ${ }^{1}$, Rong Liu ${ }^{1,2}$, Lu Lu$^{1}$, Chihong Zhang ${ }^{1}$, Lei Zhang ${ }^{1}$, Levi Yant ${ }^{3}$ and Yu Wu ${ }^{1 *}$

\begin{abstract}
Background: Heavy metal ATPases (HMAs) are responsible for $\mathrm{Cd}$ translocation and play a primary role in $\mathrm{Cd}$ detoxification in various plant species. However, the characteristics of HMAs and the regulatory mechanisms between HMAs and microRNAs in wheat (Triticum aestivum L) remain unknown.

Results: By comparative microRNA and transcriptome analysis, a total three known and 19 novel differentially expressed microRNAs (DEMs) and 1561 differentially expressed genes (DEGs) were found in L17 after Cd treatment. In H17, by contrast, 12 known and 57 novel DEMs, and only 297 Cd-induced DEGs were found. Functional enrichments of DEMs and DEGs indicate how genotype-specific biological processes responded to $\mathrm{Cd}$ stress. Processes found to be involved in microRNAs-associated $\mathrm{Cd}$ response include: ubiquitin mediated proteolysis, tyrosine metabolism, and carbon fixation pathways and thiamine metabolism. For the mRNA response, categories including terpenoid backbone biosynthesis and phenylalanine metabolism, and photosynthesis - antenna proteins and ABC transporters were enriched. Moreover, we identified 32 TaHMA genes in wheat. Phylogenetic trees, chromosomal locations, conserved motifs and expression levels in different tissues and roots under $\mathrm{Cd}$ stress are presented. Finally, we infer a microRNA-TaHMAs expression network, indicating that miRNAs can regulate TaHMAs.

Conclusion: Our findings suggest that microRNAs play important role in wheat under $\mathrm{Cd}$ stress through regulation of targets such as TaHMA2;1. Identification of these targets will be useful for screening and breeding low-Cd accumulation wheat lines.
\end{abstract}

Keywords: Wheat, RNA sequencing, microRNA, HMA

\section{Background}

Cadmium (Cd) contamination of soils is rapidly increasing from both human activities and other environmental causes [1]. Deleterious effects of $\mathrm{Cd}$ are dramatic and varied in both humans and plants. In humans, Cd ingestion leads to osteoporosis and emphysema, among other diseases [2, 3]. In plants, high $\mathrm{Cd}$ results in a wide range of biochemical and physiological disorders, inhibiting yield and quality [4]. Unfortunately, even moderate levels of $\mathrm{Cd}$ in soils can result in dramatic $\mathrm{Cd}$ accumulation in leaves

\footnotetext{
* Correspondence: wuyugood@126.com

${ }^{1}$ Chengdu Institute of Biology, Chinese Academy of Sciences, Chengdu 610041, Sichuan, China

Full list of author information is available at the end of the article
}

or grains $[5,6]$. Contamination in the food chain with $\mathrm{Cd}$ thus poses a major widespread hazard to human health [7-9]. Wheat, one of the most important worldwide crops, is grown on nearly $20 \%$ of cultivated land and serves as a staple food source for $30 \%$ of humanity [10, 11]. Previous studies indicated that compared with other cereals, wheat accumulates $\mathrm{Cd}$ primarily via roots, eventually accumulating $\mathrm{Cd}$ in edible grains $[12,13]$. Therefore, it is very important to understand wheat responses to $\mathrm{Cd}$ in order to improve wheat growth, grain quality, and human welfare.

MicroRNAs (miRNAs) are typically 21 nucleotide endogenous non-coding RNAs that play key roles in regulating gene expression post-transcriptionally, usually via cleavage or translational repression of target mRNAs

(C) The Author(s). 2019 Open Access This article is distributed under the terms of the Creative Commons Attribution 4.0 International License (http://creativecommons.org/licenses/by/4.0/), which permits unrestricted use, distribution, and 
$[14,15]$. In plants, miRNAs are involved in various physiological processes and play important roles in growth and development $[16,17]$, and numerous miRNAs participate in responses to biotic and abiotic stresses [18-21]. Deep-sequencing of developing wheat grains revealed 605 miRNAs [22] and 57 conserved miRNA families have been identified in wheat hybrid necrosis [23]. Hundreds of miRNAs were differentially expressed in leaf and roots tissues in wheat under drought stress, respectively [24]. Individual analysis of miRNAs or mRNAs have been widely reported under various stressors. However, the interplay of $\mathrm{Cd}$, regulatory miRNAs, and the transcriptome is not at all understood, so there is still an obvious gap in our understanding of their interplay in a major crop.

$\mathrm{P}_{1 \mathrm{~B}}$-ATPases, also known as heavy metal ATPases (HMAs), are proteins that use ATP to pump a wide range of cations across membranes against electrochemical gradients [25]. AtHMA2 and AtHMA4 act as pumps to efflux $\mathrm{Zn} / \mathrm{Cd}$ out of cells, playing critical role in rootshoot $\mathrm{Zn} / \mathrm{Cd}$ translocation through xylem loading [26]. The HMA3 proteins, such as AtHMA3, TcHMA3 and OsHMA3, are typically located in tonoplasts and are not regulated through $\mathrm{Zn}$ or $\mathrm{Cd}$; these proteins play a role in pumping $\mathrm{Zn}$ and/or $\mathrm{Cd}$ into vacuoles [27-29]. Much available evidence indicates that HMAs play an important role in heavy metal transmembrane transport. However, there is little understanding of HMAs in the wheat genome and their roles in wheat under Cd stress.

Therefore, to gain an understanding of the global transcriptional response and its interplay with miRNAs and HMAs in wheat during Cd stress, we used next generation transcriptome and miRNA sequencing to profile the responses of low- and high-cadmium-accumulating wheat cultivars in response to $\mathrm{Cd}$. Additionally, we paid special attention to regulatory dynamics of the HMA gene family. We further verified by qRT-PCR significantly differentially expressed of miRNAs, mRNAs and TaHMAs. Finally, we performed for gene ontology and pathway analysis, as well as depicted general functional landscapes of miRNAs-TaHMAs expression. We expect that these findings will provide a meaningful resource for the understanding the global transcriptional response to $\mathrm{Cd}$ and how these are modulated by miRNAs.

\section{Results and discussion}

Differences in cd accumulation in grains among wheat germplasms

We first screened 185 wheat germplasms for grain $\mathrm{Cd}$ levels using ICP-MS (Additional file 1: Table S1). By group, the average concentrations of $\mathrm{Cd}$ in grain decreased in the order of CMC > Chuanyu > CIMMYT, with significant differences between group averages (Fig. 1a). The tolerance limit of $\mathrm{Cd}$ in food is $0.1 \mathrm{mg} / \mathrm{kg}$ set by Ministry of Health of PRC (People's Republic of China). Thus, there were $15(28.85 \%)$ cultivars that exceeded the tolerance limit in Chuanyu group, 1 (1.79\%) in the CIMMYT group, and 56 (76.71\%) in the CMC group (Fig. 1b). Our results revealed that the average grain $\mathrm{Cd}$ concentration of four durum wheat genotypes was $0.224 \mathrm{mg} / \mathrm{kg}$, and ranged from 0.191 to 0.371 $\mathrm{mg} / \mathrm{kg}$. These four durum cultivars contained higher grain $\mathrm{Cd}$ concentrations compared with bread wheat (Additional file 2: Figure S1), in agreement with previous studies [30-32]. Previous work showed that durum wheat cultivars grown in a variety test in South Dakota contained similar average grain $\mathrm{Cd}$ concentrations $(0.22$ $\mathrm{mg} / \mathrm{kg}$, ranged from 0.13 to $0.25 \mathrm{mg} / \mathrm{kg}$ ) [33], indicating
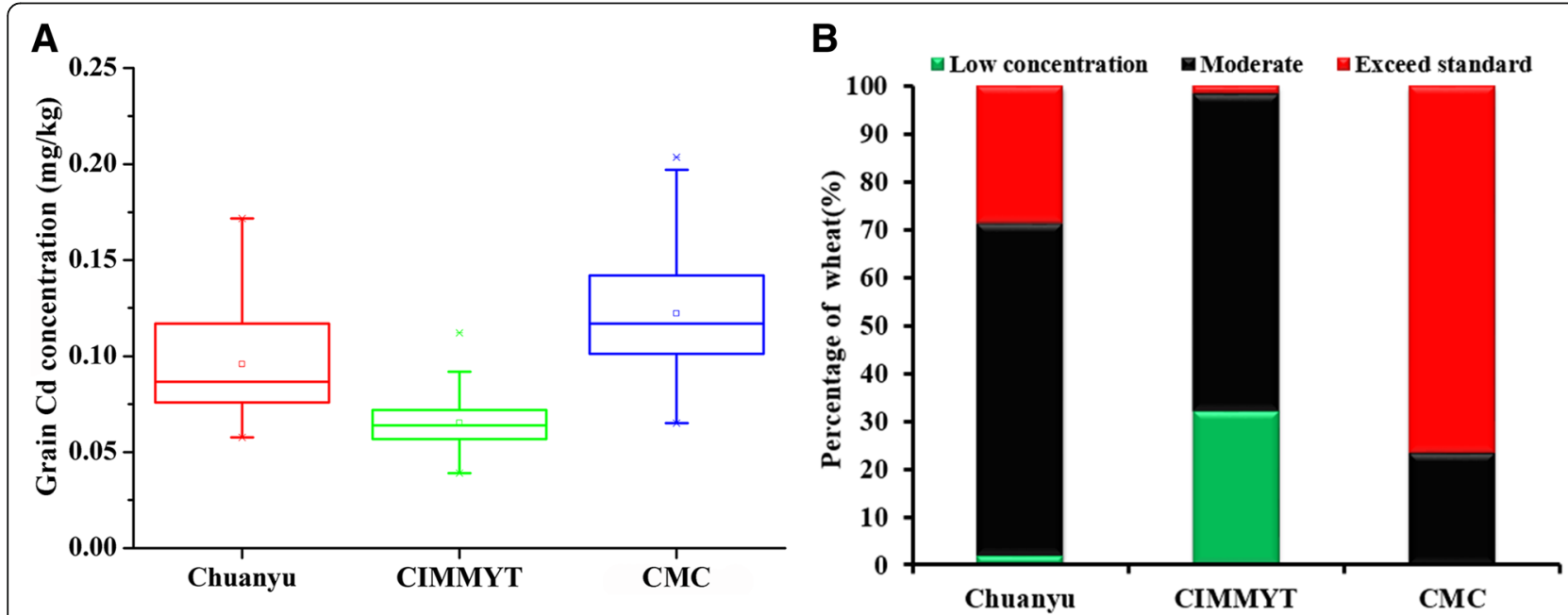

Fig. 1 a Grain Cd concentration among three major wheat groups. The Chuanyu group included 52 wheat cultivars, the CIMMYT group included 56 wheat cultivars, and the CMC group included 73 wheat cultivars. b Percentage of low Cd concentration $(\leq 0.0579$ mg/kg), moderate Cd concentrations $(<0.1 \mathrm{mg} / \mathrm{kg})$ and exceeding $\mathrm{Cd}$ concentration standard $(\geq 0.1 \mathrm{mg} / \mathrm{kg})$ wheat in each of the three groups 
that our results are good representatives of world-wide sampling.

\section{Differentially expressed miRNAs and mRNAs between L17Cd and L17CK, H17Cd and H17CK}

To illuminate the underlying molecular mechanism, we chose cultivars strongly contrasting for $\mathrm{Cd}$ content: a low-Cd-accumulating cultivar (Chuanyu 17, hereafter L17) and a high-Cd-accumulating cultivar (a Chuanyu 17 sib-line, hereafter H17) to determine differentially expressed miRNAs and mRNAs in roots. We generated 12 small RNA sequencing libraries from three biological replicates each for $\mathrm{L} 17$ and $\mathrm{H} 17$ roots under control and $\mathrm{CdCl}_{2}$ treatment and sequenced them on an Illumina HiSeq TM 2500. The major characteristics of these libraries are summarized in Table 1. Between 18.8 and 32.4 million total raw reads per library were obtained. After quality filtration, between 12.6 and 20.4 million clean reads were obtained per replicate.

After comparing the distributions of miRNA expression values in the samples after normalization and correlation analysis of miRNA expression levels between samples and samples (Additional file 2: Figure S2), we next performed miRNA expression analysis and found many clear contrasts in miRNA expression between genotypes and groups. Two miRNAs were upregulated in L17Cd (treated with $100 \mu \mathrm{M} \mathrm{CdCl}_{2}$ for $24 \mathrm{~h} \mathrm{Cd}$ ) and one was downregulated compared with $\mathrm{L} 17 \mathrm{CK}\left(\mathrm{CdCl}_{2}\right.$-free). In the high-Cd-accumulating genotype (H17), 12 miRNAs in $\mathrm{H} 17 \mathrm{Cd}$ (treated with $100 \mu \mathrm{M} \mathrm{CdCl}$ for $24 \mathrm{~h} \mathrm{Cd}$ ) were downregulated compared with $\mathrm{H} 17 \mathrm{CK}\left(\mathrm{CdCl}_{2}\right.$-free $)$ (Table 2). Regarding novel miRNAs in L17, 10 miRNAs were upregulated and nine were downregulated in L17Cd compared with L17CK, while in H17, 49 miRNAs were upregulated and eight were downregulated in H17Cd compared with H17CK (Table 3). In addition, one uniquely expressed miRNA between $\mathrm{L} 17 \mathrm{Cd}$ and L17CK, one uniquely expressed miRNA between $\mathrm{H} 17 \mathrm{Cd}$ and $\mathrm{H} 17 \mathrm{CK}$, and two expressed miRNAs were commonly expressed. These results indicated that taemiR9664-3p and tae-miR159a were upregulated in L17Cd group compared with that in L17CK group, while they were downregulated in $\mathrm{H} 17 \mathrm{Cd}$ group compared with that in H17CK group. Moreover, according to our RNA sequencing data, the putative target mRNAs (such as Traes_7BL_66C9AAA60, Traes_1BS_1E568BC28, Traes_ 5DL_BDE25A669, Traes_2DS_47DDC83DC and Traes_ 5BS_C3B48D8CC) for upregulated tae-miR9664-3p and the putative target mRNAs (such as Traes_3AL F09C7CA19, Traes_7AS_2831C6473, Traes_7DS_0FFF2 FB02 and Traes_4AL_03723C140) for upregulated taemiR159a were downregulated in L17Cd group compared with that in L17CK group. Previous work demonstrated that tae-miR-9664 was expressed in the flag leaf and developing seed of wheat (Triticum aestivum L.) [34]. TaemiR159a played positive roles in wheat response to Puccinia striiformis f.sp.tritici through the regulation of TaMyb3 expression [35]. Our results suggested that contrasting Cd-accumulating ability of L17 and $\mathrm{H} 17$ might associated with the up-regulation or down-regulation between tae-miR9663-3p, tae-miR159a and their target genes. We also found that tae-miR9774 was uniquely down-regulated between L17Cd and L17CK, while taemiR398 was specifically down-regulated between $\mathrm{H} 17 \mathrm{Cd}$ and $\mathrm{H} 17 \mathrm{CK}$. A previous study demonstrated that tae-miR9774 was expressed in wheat leaves, stems, roots and young spikes [36]. miR398 was induced by UVB light and heat stress in Arabidopsis but was inhibited by ABA, salinity, cold, and oxidative stress [37, 38]. miR398 can regulate copper superoxide dismutase (CSD) 1 and 2 that were both induced by salinity treatment $[37,39]$. These results suggested that miRNAs could participate in the growth and development of wheat and also function in wheat response to both biotic and abiotic stress. To our knowledge, this is the first study to identify differentially expressed miRNAs in response to $\mathrm{Cd}$ accumulation in multiple wheat genotypes contrasting for $\mathrm{Cd}$ accumulation.

After checking the distributions of expression values after normalization and correlation analysis of expression levels between samples (Additional file 2: Figure S3), we next performed differential expression analysis of mRNA transcripts. In the low-Cd-accumulating genotype (L17), a total of 1561 mRNAs were detected to be

Table 1 Read statistics in small RNA libraries by genotype

\begin{tabular}{lllll}
\hline Type & L17CK & L17Cd & H17CK & H17Cd \\
\hline Raw reads & $29,634,338 \pm 12,235,540$ & $23,765,742 \pm 5,243,896$ & $18,786,733 \pm 2,145,550$ & $32,365,435 \pm 7,244,882$ \\
Clean reads & $20,430,195 \pm 8,138,193$ & $14,922,483 \pm 7,059,238$ & $12,556,082 \pm 1,740,984$ & $19,285,729 \pm 5,444,137$ \\
Unique clean reads & $3,232,322 \pm 981,148$ & $2,426,636 \pm 1,239,154$ & $2,313,643 \pm 314,127$ & $2,580,979 \pm 647,449$ \\
Wheat genome-matched read (redundant) & $17,858,724 \pm 7,263,840$ & $12,629,611 \pm 5,018,126$ & $8,991,791 \pm 1,289,534$ & $14,299,057 \pm 3,343,128$ \\
Wheat genome-matched read (unique) & $2,180,300 \pm 664,560$ & $1,604,078 \pm 683,985$ & $1,313,826 \pm 142,562$ & $1,481,392 \pm 264,618$ \\
Wheat genome-matched read (redundant \%) & $87.14 \pm 1.14$ & $86.43 \pm 5.95$ & $71.61 \pm 2.78$ & $74.81 \pm 3.80$ \\
Wheat genome-matched read (unique \%) & $67.45 \pm 0.56$ & $67.82 \pm 5.18$ & $56.93 \pm 1.82$ & $58.12 \pm 4.71$ \\
\hline
\end{tabular}

In each wheat samples, data is shown as the mean of the three libraries generated \pm standard deviation 
Table 2 Known miRNAs identified in the 12 wheat sRNA libraries

\begin{tabular}{|c|c|c|c|c|c|}
\hline Comparison & miRNA family & miRNA_id & log2FoldChange & $P$ & Down \\
\hline \multirow[t]{3}{*}{ L17Cd/L17CK } & miR159a & tae-miR159a & 0.87 & $3.347 \mathrm{E}-05$ & Up \\
\hline & miR9664 & tae-miR9664-3p & 0.53 & 0.0427885 & Up \\
\hline & miR9774 & tae-miR9774 & -2.29 & 0.0279111 & Down \\
\hline \multirow[t]{12}{*}{$\mathrm{H} 17 \mathrm{Cd} / \mathrm{H} 17 \mathrm{CK}$} & miR159a & tae-miR159a & -1.04 & $5.42 \mathrm{E}-11$ & Down \\
\hline & miR167 & tae-miR167a & -1.47 & 0.0379267 & Down \\
\hline & mi395 & tae-miR395b & -2.40 & 0.0038538 & Down \\
\hline & miR397 & tae-miR397-5p & -1.50 & 0.019139 & Down \\
\hline & miR398 & tae-miR398 & -0.99 & 0.0250389 & Down \\
\hline & miR5048 & tae-miR5048-5p & -0.91 & 0.0247939 & Down \\
\hline & miR7757 & tae-miR7757-5p & -1.37 & 0.0001656 & Down \\
\hline & miR9662 & tae-miR9662a-3p & -1.10 & 7.92E-06 & Down \\
\hline & miR9664 & tae-miR9664-3p & -0.68 & 0.0203362 & Down \\
\hline & miR9669 & tae-miR9669-5p & -1.50 & 0.0258714 & Down \\
\hline & miR9674 & tae-miR9674b-5p & -0.92 & 0.0109898 & Down \\
\hline & miR9778 & tae-miR9778 & -1.30 & $1.11 \mathrm{E}-07$ & Down \\
\hline
\end{tabular}

differentially expressed with fold change $>2.0, P<0.05$ (volcano plot filtering) and FDR $<0.05$ (Additional file 2: Figure S4, Fig. 2a). Among them, 1208 and 353 mRNAs were upregulated and downregulated (fold change $>2.0$, $P<0.05$ and FDR $<0.05)$ in $\mathrm{L} 17$ roots compared with controls, respectively. In the high-Cd-accumulating genotype (H17), a total of 297 mRNAs were found to be differentially expressed with a fold change $>2.0, P<0.05$ (volcano plot filtering) and FDR $<0.05$ (Additional file 2: Figure S4, Fig. 2b). Among them, 204 and 93 mRNAs were upregulated and downregulated (fold change $>2.0$, $P<0.05$ and FDR $<0.05)$ in $\mathrm{H} 17$ roots compared with controls, respectively.

\section{Verification of differentially expressed miRNAs and mRNAs}

To confirm the results of the differential expression analyses, eight $\mathrm{Cd}$-induced miRNAs and six $\mathrm{Cd}$-induced mRNAs were selected for qRT-PCR expression analysis according to their fold change and $\mathrm{GO}$ analysis. For microRNAs, compared with L17CK group, tae-miR159a, tae-miR9446-3P, microRNA-2B_39534* were increased in L17Cd group, while tae-miR9774, microRNA-2B_28100* were decreased (Fig. 3a). Compared with H17CK group, microRNA-2B_28100* was increased in H17Cd group, while tae-miR159a, tae-miR395b, tae-miR398, taemiR7757-5P and tae-miR9664-3P microRNA-2B_28100* (Fig. 3a). For mRNAs, compared with L17CK group, Traes_4AS_8944253DC was increased in L17Cd group, while Traes_2AL_E5172BD9D was decreased (Fig. 3b). Compared with H17CK group, Traes_4AS_8944253DC, Traes_5AL_B4E8A3115 and Traes_2BS_1484A7516 were increased in H17Cd group, while Traes_6DS_C59B6322F and Traes_2DS_01A0E5F7A (Fig. 3b). Hence, the qRTPCR data verified the small RNA and RNA sequencing results.

\section{GO and KEGG enrichment analysis}

It is well known that miRNAs play essential roles in plant growth and development and also control plant responses to biotic and abiotic stresses by regulating targeted gene expression [23, 40]. Therefore, Gene Ontology (GO) analysis of targeted mRNAs of miRNAs can real the role of differentially expressed miRNAs. Enriched GO categories between L17Cd and L17CK groups, $\mathrm{H} 17 \mathrm{Cd}$ and $\mathrm{H} 17 \mathrm{CK}$ groups, according to the gene number enriched in GOs, the top 10 biological process (BP), molecular function (MF) and cellular component $(\mathrm{CC})$ for downregulated miRNAs and upregulated miRNAs were shown in (Additional file 2: Figure S5). Overlaying gene sets (Additional file 2: Figure S6) revealed that down-regulated miRNAs in L17 consisted of genes involved in ubiquitin-dependent protein catabolic processes and cell wall organization, whereas upregulated miRNAs in L17 consist of those involved in protein glycosylation and oxidoreductase activity. Downregulated miRNAs in $\mathrm{H} 17$ consist of those involved in vesicle-mediated transport, and response to heat, whereas up-regulated miRNAs in H17 consist of those involved in photosynthetic electron transport chain and iron ion binding.

For mRNAs, GOs between L17Cd and L17CK groups, $\mathrm{H} 17 \mathrm{Cd}$ and $\mathrm{H} 17 \mathrm{CK}$ groups, according to the gene number enriched in GOs, the top $10 \mathrm{BP}$, MF and CC for upregulated mRNAs and downregulated mRNAs were shown in (Additional file 2: Figure S7). Based on the 
Table 3 Summary of newly identified novel miRNAs in the 12 wheat sRNA libraries

\begin{tabular}{|c|c|c|c|c|c|c|}
\hline Comparison & miRNA_id & $\log 2$ (Fold Change) & $P$ & Down & Mature sequence $\left(5^{\prime}-3^{\prime}\right)$ & Length \\
\hline \multirow[t]{19}{*}{ L17Cd/L17CK } & 2B_20696 ${ }^{\mathrm{a}}$ & 1.69 & 0.0270291 & Up & CUCGCCGGUCGCGCGUCCUCC & 21 \\
\hline & 2B_21940 & 2.33 & 0.0462198 & Up & AUGACACGGGGAGGUAGC & 18 \\
\hline & 2B_39534 ${ }^{\mathrm{a}}$ & 6.11 & 0.0176417 & Up & AGGCUGGAGGAACGUAGG & 18 \\
\hline & $2 B \_40590^{\mathrm{a}}$ & 2.00 & 0.0383352 & Up & GUGUGGAGCAAAAGGGUG & 18 \\
\hline & 4B_10717 $\mathrm{F}^{\mathrm{a}}$ & 4.18 & 0.0021475 & Up & CCCCGGGUGCGAGCUCCU & 18 \\
\hline & $4 B \_12465^{\mathrm{a}}$ & 5.45 & 0.0221443 & Up & CCUCCUGGUGAGCCUGCG & 18 \\
\hline & $4 \mathrm{~B} \_4791^{\mathrm{a}}$ & 1.96 & 0.0091409 & Up & UGGGGUUGUGGCAAUGGCC & 19 \\
\hline & 4B_519 & 2.40 & 0.0050086 & Up & AAUCUGGUUGUCGCCUCC & 18 \\
\hline & 4B_5711 ${ }^{\mathrm{a}}$ & 4.20 & 0.0024414 & Up & GUGAAGAGACAUGGAGGU & 18 \\
\hline & 4B_9306 & 1.13 & 0.0050015 & Up & UAGAAUGGCUGGUGCUAUGGA & 21 \\
\hline & 2B_19887 ${ }^{\mathrm{a}}$ & -4.18 & 0.0352225 & Down & ACGGACCGCGCUACUACUAUAAGU & 24 \\
\hline & 2B_22021 ${ }^{\mathrm{a}}$ & -1.39 & 0.016954 & Down & AUGGAAGACGUGGUAGCC & 18 \\
\hline & 2B_28100 & -1.27 & 0.0412731 & Down & AGGUAGAGAGGAAGGUGGG & 19 \\
\hline & $2 B \_33543^{\mathrm{a}}$ & -3.40 & 0.0480069 & Down & CUCGCCGGAGGAGCGUGC & 18 \\
\hline & $2 B \_38598^{\mathrm{a}}$ & -2.15 & 0.0214449 & Down & CUGCUGGUGCUGUAGCCCU & 19 \\
\hline & 4B_17875 & -4.36 & 0.0138807 & Down & UUCCAAUUUACUCGUCGUGGU & 21 \\
\hline & 4B_4344 & -2.68 & 0.0327648 & Down & GGUGUGGACCAGGUUUCC & 18 \\
\hline & $4 \mathrm{~B} \_87^{\mathrm{a}}$ & -4.35 & 0.0106819 & Down & CGCGUGCAGGAUGAAGCC & 18 \\
\hline & 4B_8797 & -2.08 & 0.0458935 & Down & ACUCACUCUGUAAACUAAUAUAAG & 24 \\
\hline \multirow[t]{25}{*}{$\mathrm{H} 17 \mathrm{Cd} / \mathrm{H} 17 \mathrm{CK}$} & 2B_19349 & 4.00 & 1.83E-06 & Up & AAACAGUCUGUAAAGCCCC & 19 \\
\hline & 2B_20999 & 2.42 & $1.06 \mathrm{E}-14$ & Up & CUACGGGGGAAAGCAGGG & 18 \\
\hline & 2B_21614 & 2.02 & 0.0023063 & Up & UGUUGAAGUAGCUGGAAC & 18 \\
\hline & 2B_21995 & 1.41 & 0.002425 & Up & CGGAGAGGGGAGUGAAGU & 18 \\
\hline & 2B_22021 ${ }^{\mathrm{a}}$ & 2.93 & $9.43 \mathrm{E}-27$ & Up & AUGGAAGACGUGGUAGCC & 18 \\
\hline & 2B_22879 & 3.19 & 0.0120242 & Up & CGGCCAUCGUGAGGUUAGUGC & 21 \\
\hline & 2B_25173 & 2.36 & 0.0000977 & Up & GACUUUGAUCCAGAGAUCA & 19 \\
\hline & 2B_27180a & 1.86 & 0.0252563 & Up & GUUGGUCGGGAGUUCGAUCCU & 21 \\
\hline & $2 B \_28100^{\mathrm{a}}$ & 1.76 & 4.01E-07 & Up & AGGUAGAGAGGAAGGUGGG & 19 \\
\hline & 2B_28714 & 1.46 & 0.0001773 & Up & AUGAGAGGACGGACAAGG & 18 \\
\hline & 2B_29589 ${ }^{\mathrm{a}}$ & 1.98 & 0.0040311 & Up & GAGUUUGGGAGUCUGUGUGU & 20 \\
\hline & 2B_32148 & 1.66 & 0.0364154 & Up & UCAGUGCAAUCCCUCUGGAAU & 21 \\
\hline & $2 \mathrm{~B} \_33858^{\mathrm{a}}$ & 1.58 & 0.0226808 & Up & CGUGCCAGCAGCCGCGGA & 18 \\
\hline & $2 \mathrm{~B} \_37122^{\mathrm{a}}$ & 1.33 & 0.0002491 & Up & UCUGGUGGAUGCCUUGGG & 18 \\
\hline & $2 B \_37173^{\mathrm{a}}$ & 1.15 & 0.0031618 & Up & AGAGGGUGGUGGAGUUUCU & 19 \\
\hline & 2B_38197 $\mathrm{a}^{\mathrm{a}}$ & 5.03 & $1.57 \mathrm{E}-10$ & Up & UUGUUGGAUAUGUUGGUU & 18 \\
\hline & 2B_40139 & 2.06 & 0.0018813 & Up & GUGGAUGCCUUGGCGAUC & 18 \\
\hline & 2B_40352 ${ }^{\mathrm{a}}$ & 2.74 & 0.0074044 & Up & CAAUGGUAGAGCAGAGGC & 18 \\
\hline & $2 B \_42257^{\mathrm{a}}$ & 1.93 & 0.0031222 & Up & GGCGGCGGACGGGUGAGC & 18 \\
\hline & $2 B \_42905^{a}$ & 0.91 & 0.0142497 & Up & GAGGUGCUGCAUGGCUGG & 18 \\
\hline & $2 B \_43522^{a}$ & 1.91 & 0.0003365 & Up & UGAGGUUUGAGAGGGGCU & 18 \\
\hline & 4B_10203 & 1.29 & 0.0034214 & Up & GAGGCGAUGAAGGACGGG & 18 \\
\hline & 4B_10621 $1^{\mathrm{a}}$ & 1.88 & 0.0391309 & Up & GGGUGAAGCUGUGGCGCG & 18 \\
\hline & 4B_10870 & 2.94 & $6.11 \mathrm{E}-06$ & Up & UGGUUCUGUAUGGAAGUG & 18 \\
\hline & $4 B \_12067^{\mathrm{a}}$ & 1.14 & 0.0151284 & Up & AUGAACGCUAGCGGGAGG & 18 \\
\hline
\end{tabular}


Table 3 Summary of newly identified novel miRNAs in the 12 wheat sRNA libraries (Continued)

\begin{tabular}{|c|c|c|c|c|c|c|}
\hline Comparison & miRNA_id & log2 (Fold Change) & $P$ & Down & Mature sequence $\left(5^{\prime}-3^{\prime}\right)$ & Length \\
\hline & 4B_12312 & 3.38 & 0.0022606 & Up & GAGUGGUCGAGCGGGACGG & 19 \\
\hline & 4B_13500 & 2.41 & 0.0044361 & Up & GGUUCUGUAUGGAAGUGG & 18 \\
\hline & 4B_13640 $0^{\mathrm{a}}$ & 1.37 & 0.0041213 & Up & AGGUCUGGGUUCGAGUCC & 18 \\
\hline & 4B_1373 & 2.42 & 0.0134111 & Up & GCUUGUCGCUGGUUUGAG & 18 \\
\hline & 4B_15373 & 0.92 & 0.0420051 & Up & GUGGCUCCACGUGGGCGGGC & 20 \\
\hline & 4B_15496 ${ }^{\mathrm{a}}$ & 1.63 & 0.0036066 & Up & UGCGGGAUGGAGCAGUCG & 18 \\
\hline & 4B_15927 & 2.37 & $2.51 \mathrm{E}-08$ & Up & AUGCUAGUCGAGCGGAUA & 18 \\
\hline & $4 B \_16269^{a}$ & 3.22 & $3.8 \mathrm{E}-11$ & Up & AGGUCGACGGUUCGAAUCC & 19 \\
\hline & 4B_16562 & 1.34 & 0.0445484 & Up & CGGAGGUCGCGGGUUCGA & 18 \\
\hline & 4B_16625 & 1.94 & 0.0000161 & Up & UCCUGGGGUUGGAGAAGG & 18 \\
\hline & 4B_18394 & 1.24 & 0.007484 & Up & GCGGCGAGAGCGGGUCGCU & 19 \\
\hline & 4B_1943 & 1.85 & 0.0000179 & Up & ACAGUGGAUGCCUUGGCA & 18 \\
\hline & $4 B \_2104^{a}$ & 1.61 & 0.0136994 & Up & AAGCCUCUGGCGAAUGGG & 18 \\
\hline & 4B_2324 ${ }^{\mathrm{a}}$ & 3.28 & 0.0050367 & Up & GAUUUAUCGGGGAAGGAU & 18 \\
\hline & $4 \mathrm{~B} \_2847^{\mathrm{a}}$ & 3.91 & 0.0001901 & Up & GAGGAAGGUGGAUGACGG & 18 \\
\hline & 4B_4039 & 3.73 & 0.0200414 & Up & GAACGGAAGUUGGGGGCG & 18 \\
\hline & $4 B \_4609^{a}$ & 3.46 & 0.0000312 & Up & GAUCUUGAUGUGGUGUGCA & 19 \\
\hline & $4 B \_4703^{a}$ & 2.74 & $1.23 \mathrm{E}-07$ & Up & GUGAUUCAGCGGCGGACG & 18 \\
\hline & 4B_5897 & 1.35 & 0.0138282 & Up & AGGAGGGUGGGGAUGAGGU & 19 \\
\hline & 4B_5947 & 2.75 & 0.0124835 & Up & GAGUGGUCGAGCGGGACGG & 19 \\
\hline & 4B_6530 ${ }^{\mathrm{a}}$ & 2.05 & $3.8 \mathrm{E}-10$ & Up & GCUCGUGUCGUGAGCUGG & 18 \\
\hline & 4B_841 ${ }^{\mathrm{a}}$ & 1.64 & 0.0087137 & Up & GAAGGUCGUGGGUUCGUG & 18 \\
\hline & $4 \mathrm{~B} \_88^{\mathrm{a}}$ & 3.20 & 0.0471916 & Up & AGACGUUUCCGUCGACUACGAAA & 23 \\
\hline & 4B_974 ${ }^{\mathrm{a}}$ & 1.79 & 0.0009387 & Up & UAUCGUGGGUUCGAAUCC & 18 \\
\hline & 2B_19630 & -1.52 & 0.0134799 & Down & CAGCCUGCCAACCCUGGG & 18 \\
\hline & 2B_23520 & -5.38 & $3.24 \mathrm{E}-07$ & Down & GCUUCGGCGGUAUCCUCAU & 19 \\
\hline & $2 B \_27285^{\mathrm{a}}$ & -1.72 & 0.0294342 & Down & GAUGGGUCUCUGAGGGAU & 18 \\
\hline & $2 B \_40624^{a}$ & -1.93 & 0.0212515 & Down & UGGCGAUGAGGAACGGAA & 18 \\
\hline & 2B_40888 ${ }^{\mathrm{a}}$ & -2.07 & 0.0151122 & Down & GGCGGAGGCUGCGGGUUU & 18 \\
\hline & 4B_11590 & -2.33 & 0.0093351 & Down & GAGGGGUGUUGAUUUUCCGG & 20 \\
\hline & 4B_7298 & -1.77 & 0.0132316 & Down & GAGGGGUGUUGAUUUUCCGG & 20 \\
\hline & 4B_7309 & -2.87 & 0.0008129 & Down & GAACGGAAGUUGGGGGCG & 18 \\
\hline
\end{tabular}

${ }^{\text {a Highly conserved miRNAs }}$

results of Venn diagrams, we found that up-regulated mRNAs in L17 prefer to cell wall macromolecule catabolic process and response to stress, whereas downregulated mRNAs in L17 prefer to cell wall organization and DNA replication; up-regulated mRNAs in $\mathrm{H} 17$ prefer to photosynthesis and nicotianamine biosynthetic process, whereas down-regulated mRNAs in H17 prefer to defense response and metal ion transport (Additional file 2: Figure S6).

Analysis of pathway enrichment for miRNAs between L17Cd and L17CK groups revealed a diverse set of processes responsive to $\mathrm{Cd}$ (Fig. 4a, b). Among these pathways, starch and sucrose metabolism was the most prominent pathway targeted by downregulated miNAs (Fig. 4a), whereas glycolysis / gluconeogenesis was the top enriched pathway for up-regulated miRNAs (Fig. $4 \mathrm{~b}$ ). In the $\mathrm{H} 17 \mathrm{Cd}$ and $\mathrm{H} 17 \mathrm{CK}$ contrasts we found that carbon metabolism was the most prominent pathway targeted by downregulated miNAs (Fig. 4c), whereas photosynthesis was the most prominent pathway targeted by upregulated miNAs (Fig. 4d). For mRNAs differentially regulated between $\mathrm{L} 17 \mathrm{Cd}$ and L17CK groups, the top 10 pathways associated with up- or down-regulated mRNAs are given in Fig. 5. Among 
A

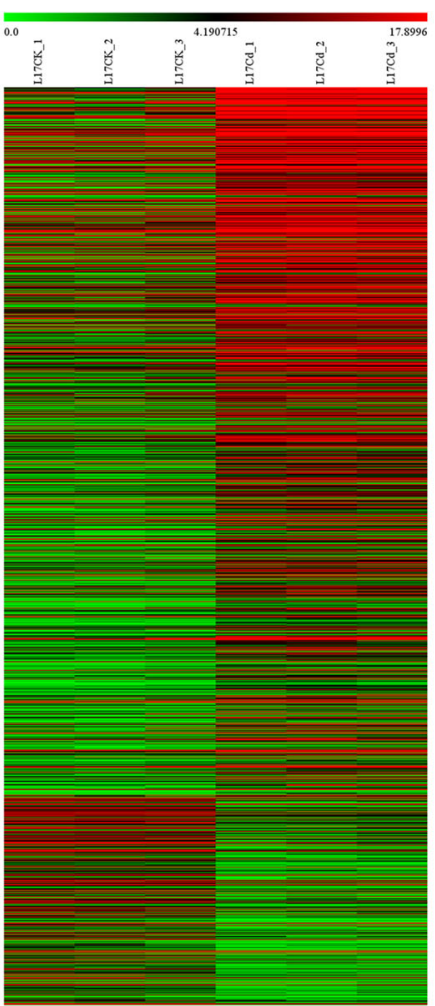

B
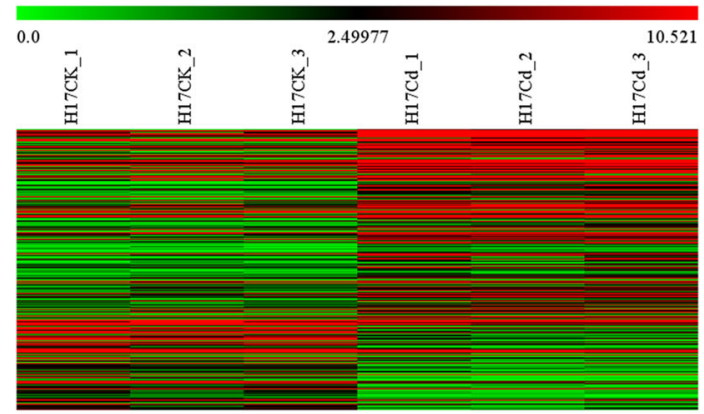

Fig. 2 Differentially expressed mRNAs. a Heat map of differentially expressed mRNA with fold changes $\geq 2.0, P<0.05$ and false discovery rate $(F D R)<0.05$ between L17Cd and L17CK. $\mathbf{b}$ Heat map of differentially expressed mRNA with fold changes $\geq 2.0, P<0.05$ and FDR $<0.05$ between $\mathrm{H} 17 \mathrm{Cd}$ and $\mathrm{H} 17 \mathrm{CK}$
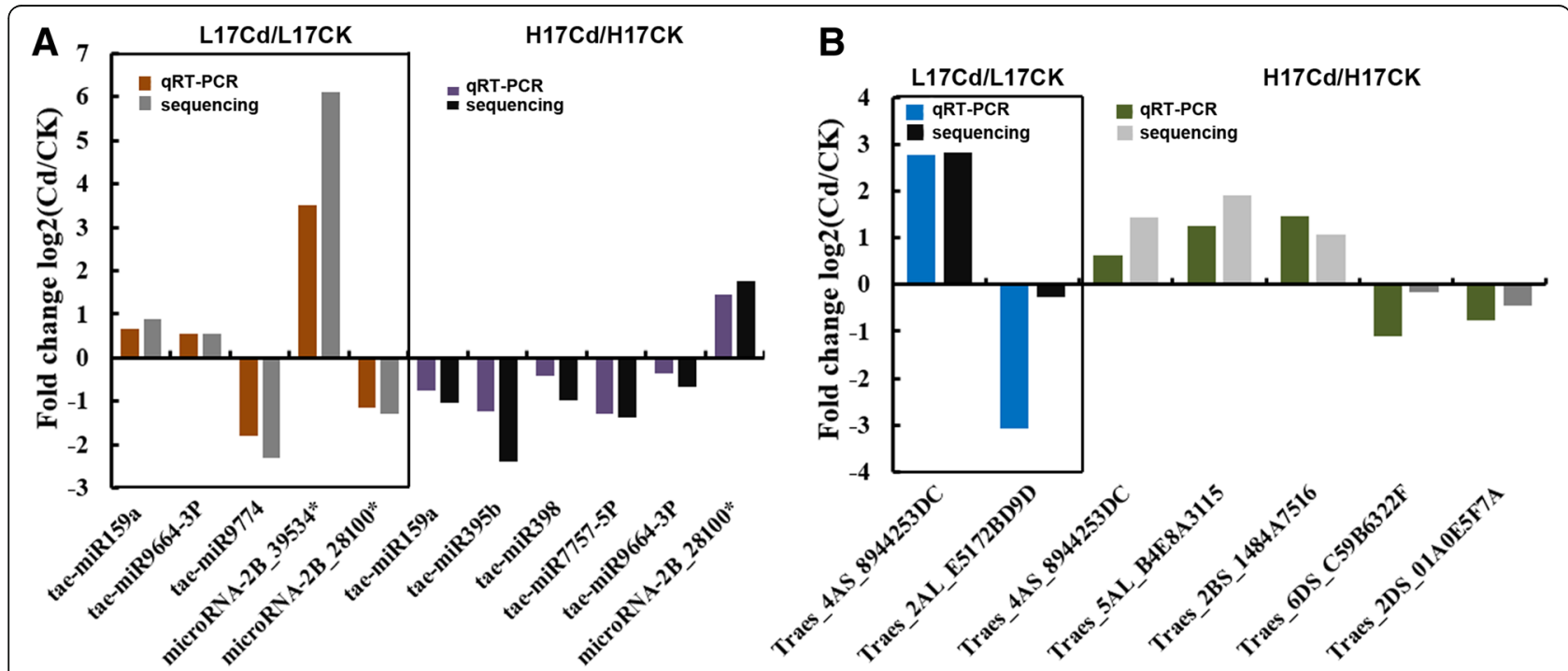

Fig. 3 qRT-PCR verification of differential expression analysis. a Relative expression levels of five miRNAs shown comparing L17Cd and L17CK (left) and six miRNAs are shown comparing H17Cd and H17CK (right). The heights of the columns stand for the fold changes (log2 transformed) computed from both qRT-PCR and small RNA sequencing data. b Relative expression levels of two mRNAs, comparing L17Cd and L17CK (left) and five mRNAs, shown comparing $\mathrm{H} 17 \mathrm{Cd}$ and $\mathrm{H} 17 \mathrm{CK}$ (right). The heights of the columns stand for the fold changes (log2 transformed) computed from the qRT-PCR and RNA sequencing data. A good correlation of qRT-PCR results and RNA sequencing data are shown through comparing such two results in both cases 


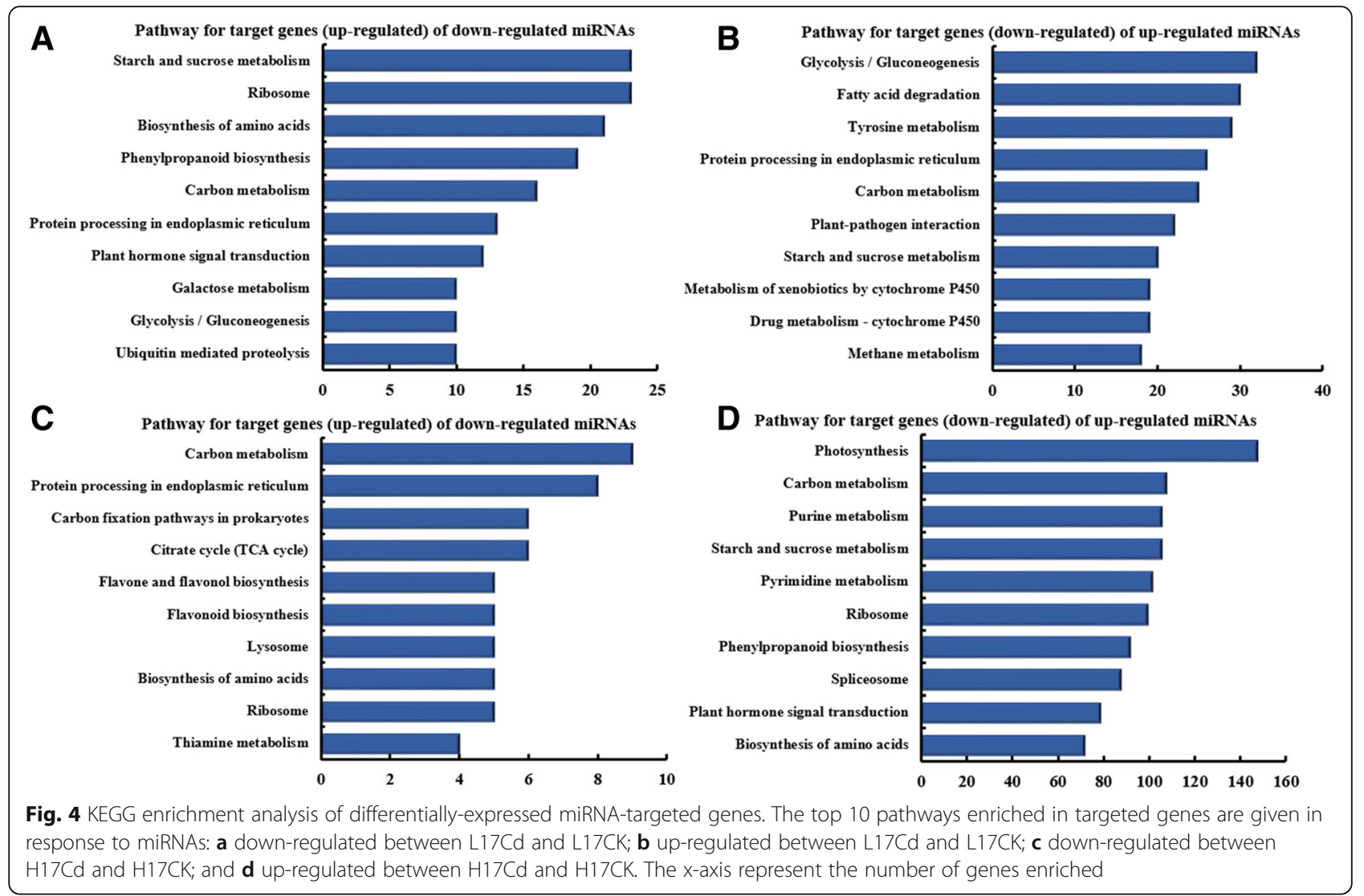

these pathways, phenylpropanoid biosynthesis was the top pathway in upregulated mRNAs (Fig. 5a), whereas starch and sucrose metabolism was the top one in downregulated mRNAs (Fig. 5b). Pathways enriched in the $\mathrm{H} 17 \mathrm{Cd}$ and $\mathrm{H} 17 \mathrm{CK}$ groups are given in Fig. $5 \mathrm{c}$, where glutathione metabolism was most prominent. On the other hand, phenylpropanoid biosynthesis was the most prominent in down-regulated mRNAs (Fig. 5d).

Our results are complement previous work in other systems. Feng et al. [41] found enrichment of genes differentially-expressed in response to $\mathrm{Cd}$ in two sweet sorghum genotypes. Enriched in the high $\mathrm{Cd}$ accumulating sweet sorghum genotype were genes that Sb04g033320, Sb04g033330, and Sb01g012440. Enriched in the low $\mathrm{Cd}$-accumulating sweet sorghum genotypes were genes that Sb02g006960, Sb01g043400, and Sb10g022390. Other studies have indicated that transporters for essential elements such as $\mathrm{Fe}^{2+}, \mathrm{Zn}^{2+}$ and $\mathrm{Ca}^{2+}$ are involved in $\mathrm{Cd}$ uptake and transport [42]. Numerous heavy metal transporters, such as ATPbinding cassette transporters ( $\mathrm{ABC}$ ), natural resistanceassociated macrophage proteins (Nramp) and heavy metal ATPases (HMA) participate in the acquisition, distribution, and homeostasis of Cd in plants [27, 43, 44].
The MAPK signaling pathway is also part of the miRNA-target gene network. MAPK signaling cascades are one of the most conserved pathways in plants and are known to modulate plant response to abiotic stress [45]. Taken together, these studies and ours indicate that the interaction effects of multiple miRNAs are involved in the regulation of diverse physiological systems to relieve the toxicity induced by $\mathrm{Cd}$.

\section{Genes related to transport}

Between L17Cd and L17CK groups 223 up-regulated genes and 183 down-regulated genes are classified as transporters based on functional annotation. Between H17Cd and H17CK, 162 up-regulated genes and 134 down-regulated genes were transporter genes according to the functional annotation of the differentially expressed genes (Additional file 3). Of these, Traes 2AL_9B175F3DA, Traes_2AS_95611CAD2, Traes_2DL_ D5F2272A9, Traes_2BS_3212EB7DF, Traes_7BL_39745 BF3F, Traes_7DL_44DA42073, Traes_2DL_2A1D45D02, Traes_3DL_4EE343C68, Traes_5BL_4BCE2DBEF, Traes_ 2BS_3212EB7DF, Traes_3AS_CBB5F7EB6, Traes_4DL 560FD0832, Traes_7AL_3E508AA9C, Trae_7DL_A5269 C73F, Trae_7AL_8304348B7 and Trae_7BL_A4BE6BD10 are related to metal ion. 

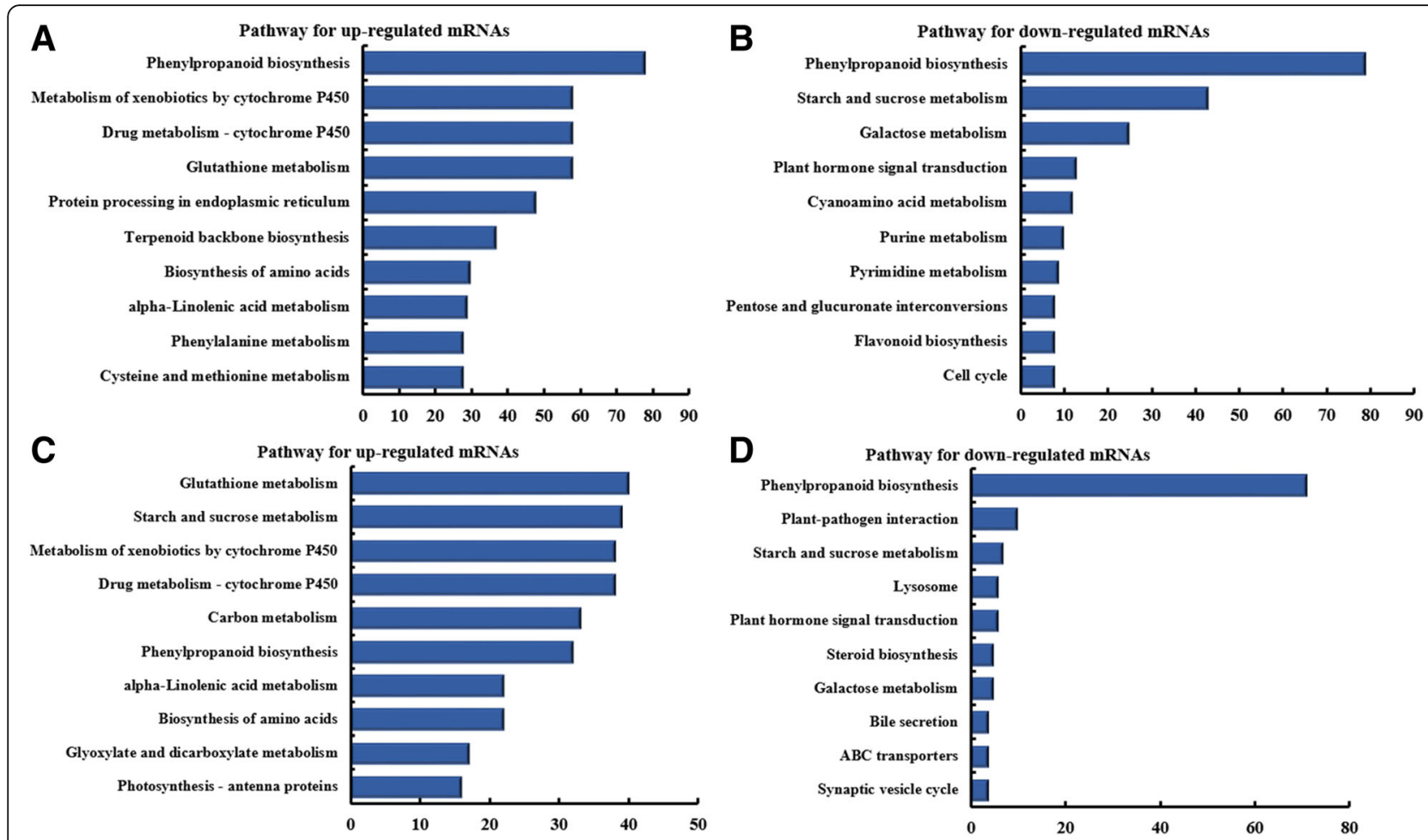

Fig. 5 KEGG pathway analysis for differentially-expressed mRNAs. The top 10 pathways enriched in differentially expressed mRNAs are given for the contrasts: $\mathbf{a}$ up-regulated between L17Cd and L17CK; $\mathbf{b}$ down-regulated between L17Cd and L17CK; $\mathbf{c}$ up-regulated between H17Cd and H17CK; and d down-regulated between $\mathrm{H} 17 \mathrm{Cd}$ and H17CK. The $\mathrm{x}$-axis represent the gene number enriched in pathways

\section{Genome-wide identification and phylogenetic analysis of HMA gene in wheat}

Through the availability of the wheat genome sequence, it is now possible to identify all the HMA family members in wheat. Here we identify a total of 32 HMA members in the wheat genome (Table 4) based on the genome sequence. We further performed a BLASTN search against the wheat expressed sequence tag (EST) using these HMAs queries to verify the existence of these wheat HMAs. Results showed that most of the TaHMAs' we found in the genome sequence were supported by EST hits. Among the supported TaHMA genes, TaHMA1;4 has the largest hits of ESTs, with 61, followed by TaHMA6;3 with 53. The lengths of these predicted TaHMA proteins varied from 419 aa to 1702 aa with the putative molecular weights $(\mathrm{Mw})$ ranging from 44.07 to $185.21 \mathrm{kDa}$ and theoretical isoelectric points (PI) ranging from 5.23 to 8.21. Eight HMA proteins are known in A.thaliana and nine in O.sativa, which range from 542 aa to 1171 aa and from 792 aa to 1060 aa, respectively [46]. Using full-length HMA protein sequences from Triticum aestivum, A.thaliana and O.sativa, we constructed a phylogentic tree to determine the phylogenetic relationship between HMA proteins from Triticum aestivum and those from other species. The HMA gene families could be divided into three groups: the $\mathrm{Cu} / \mathrm{Ca} / \mathrm{Zn} / \mathrm{Cd} / \mathrm{Co}-\mathrm{ATPases}$ group, the $\mathrm{Zn} /$ $\mathrm{Cd} / \mathrm{Pb} / \mathrm{Co}$-ATPase group, and the $\mathrm{Cu}$-ATPase group [47]. TaHMA1;1-TaHMA1;5 belong to the $\mathrm{Cu} / \mathrm{Ca} / \mathrm{Zn} /$ Cd/Co-ATPases group, TaHMA2;1-TaHMA2;6, TaHM A3;1-TaHMA3;2 belong to the $\mathrm{Zn} / \mathrm{Cd} / \mathrm{Pb} / \mathrm{Co}-\mathrm{ATPase}$ group, TaHMA4;1-TaHMA4;3, TaHMA5;1-TaHMA5;3, TaHMA6;1-TaHMA6;3, TaHMA7;1-TaHMA7;3, TaHMA 8;1-TaHMA8;3, TaHMA9;1-TaHMA9;3 belong to the $\mathrm{Cu}$-ATPase group (Fig. 6). To our knowledge, it is first genome-wide analysis of the HMA family in wheat.

\section{Chromosomal location, gene structure and conserved motif analysis of TaHMAs}

Chromosome localization analysis revealed that 31 of the TaHMA genes were distributed on $2 \mathrm{~A}, 2 \mathrm{~B}, 2 \mathrm{D}, 4 \mathrm{~A}, 4 \mathrm{D}$, $5 \mathrm{~A}, 5 \mathrm{~B}, 5 \mathrm{D}, 6 \mathrm{~A}, 6 \mathrm{~B}, 6 \mathrm{D}, 7 \mathrm{~A}, 7 \mathrm{~B}$ and 7D, of which 7A, 7B, $7 \mathrm{D}$ contained the greatest number of HMA genes, while one TaHMA did not have a corresponding chromosome (Table 4). To further understand the features of the genes of the HMA family, the exon/intron structures of TaHMA genes were analyzed. As shown in Table 1, the number of introns varied from 6 to 20. TaHMA8;3 has the largest number of introns (19), with TaHMA3;1, TaHMA3;2, TaHMA3;3, TaHMA5;1, TaHMA5;2 and TaHMA5;3 having the fewest introns (5). Although the exons varied among TaHMA genes, the most closely related members 
Table 4 Identified putative wheat HMA genes

\begin{tabular}{|c|c|c|c|c|c|c|c|c|c|c|}
\hline HMAs & $\begin{array}{l}\text { Subfamily } \\
\text { gene name }\end{array}$ & $\begin{array}{l}\text { Ensemble Wheat } \\
\text { Gene ID }\end{array}$ & Chromosome location & $\begin{array}{l}\text { Length } \\
\text { (bp) }\end{array}$ & Exons & Introns & $\begin{array}{l}\text { Amino acid } \\
\text { length (aa) }\end{array}$ & $\mathrm{Pl}$ & $\begin{array}{l}\mathrm{MW} \\
(\mathrm{kDA})\end{array}$ & $\begin{array}{l}\text { EST } \\
\text { count }\end{array}$ \\
\hline TaHMA1;1 & TaHMA1 & Trae_5AL_C89EEBE50 & $\begin{array}{l}\text { TGACv1_scaffold_375043_ } \\
\text { 5AL:69226-81,875 }\end{array}$ & 12,650 & 16 & 15 & 790 & 6.31 & 84.44 & 2 \\
\hline TaHMA1;2 & & Trae_5BL_F83C809F0 & $\begin{array}{l}\text { TGACv1_scaffold_405147_ } \\
\text { 5BL:88295-97,375 }\end{array}$ & 9081 & 13 & 12 & 480 & 8.21 & 51.96 & 2 \\
\hline TaHMA1;3 & & nd & $\begin{array}{l}\text { TGACv1_scaffold_556453_ } \\
\text { 7AL:106656-112,341 }\end{array}$ & 5686 & 8 & 7 & 419 & 6.56 & 44.07 & 20 \\
\hline TaHMA1;4 & & Trae_7BL_041308E74 & $\begin{array}{l}\text { TGACv1_scaffold_578186_ } \\
\text { 7BL:25106-34,662 }\end{array}$ & 9557 & 14 & 13 & 832 & 6.90 & 88.37 & 61 \\
\hline TaHMA1;5 & & Trae_7DL_FBCC75BBC & $\begin{array}{l}\text { TGACv1_scaffold_603768_ } \\
\text { 7DL:23146-31,623 }\end{array}$ & 8478 & 12 & 11 & 748 & 6.98 & 78.96 & 39 \\
\hline TaHMA2;1 & TaHMA2 & Trae_7DL_A5269C73F & $\begin{array}{l}\text { TGACv1_scaffold_602651_ } \\
\text { 7DL:105511-113,285 }\end{array}$ & 7742 & 10 & 9 & 1003 & 6.53 & 108.33 & 34 \\
\hline TaHMA2;2 & & Trae_7AL_8304348B7 & $\begin{array}{l}\text { TGACv1_scaffold_556712_ } \\
\text { 7AL:90458-108,176 }\end{array}$ & 17,719 & 9 & 8 & 985 & 6.29 & 106.46 & 21 \\
\hline TaHMA2;3 & & Trae_7BL_A4BE6BD10 & $\begin{array}{l}\text { TGACv1_scaffold_579416_ } \\
\text { 7BL:14127-24,069 }\end{array}$ & 9282 & 10 & 9 & 1023 & 6.51 & 110.32 & 37 \\
\hline TaHMA2;4 & & Trae_7BL_0CF58CF4E & $\begin{array}{l}\text { TGACv1_scaffold_577252_ } \\
\text { 7BL:118443-123,897 }\end{array}$ & 5455 & 9 & 8 & 766 & 7.83 & 81.09 & 3 \\
\hline TaHMA2;5 & & Trae_7AL_6AE850114 & $\begin{array}{l}\text { TGACv1_scaffold_557470_ } \\
\text { 7AL:61198-66,965 }\end{array}$ & 5768 & 10 & 9 & 939 & 7.11 & 99.55 & 6 \\
\hline TaHMA2;6 & & Trae_7DL_EED4BED66 & $\begin{array}{l}\text { TGACv1_scaffold_603863_ } \\
\text { 7DL:43200-49,689 }\end{array}$ & 5572 & 8 & 7 & 800 & 6.44 & 84.37 & 6 \\
\hline TaHMA3;1 & TaHMA3 & Trae_5BL_D6C3DC326 & $\begin{array}{l}\text { TGACv1_scaffold_404346_ } \\
\text { 5BL:222670-226,813 }\end{array}$ & 4108 & 6 & 5 & 829 & 6.05 & 86.82 & 3 \\
\hline TaHMA3;2 & & nd & $\begin{array}{l}\text { TGACv1_scaffold_375473_ } \\
\text { 5AL:29654-33,170 }\end{array}$ & 3517 & 6 & 5 & 816 & 6.29 & 85.66 & 2 \\
\hline TaHMA3;3 & & nd & $\begin{array}{l}\text { TGACv1_scaffold_435190_ } \\
\text { 5DL:5098-8725 }\end{array}$ & 3628 & 6 & 5 & 853 & 6.12 & 89.09 & 3 \\
\hline TaHMA4;1 & TaHMA4 & Trae_6DS_26C5A0A44 & $\begin{array}{l}\text { TGACv1_scaffold_543118_ } \\
\text { 6DS:56997-63,045 }\end{array}$ & 6049 & 10 & 9 & 687 & 6.01 & 73.46 & 30 \\
\hline TaHMA4;2 & & Trae_6BS_A8B960E60 & $\begin{array}{l}\text { TGACv1_scaffold_515718_ } \\
6 \text { BS:7391-13,506 }\end{array}$ & 6116 & 9 & 8 & 980 & 5.44 & 105.63 & 29 \\
\hline TaHMA4;3 & & Trae_6AS_6F306F27E & $\begin{array}{l}\text { TGACv1_scaffold_485988_ } \\
\text { 6AS:25642-29,385 }\end{array}$ & 3744 & 7 & 6 & 687 & 6.01 & 73.47 & 25 \\
\hline TaHMA5;1 & TaHMA5 & Trae_2DL_51FF05F66 & $\begin{array}{l}\text { TGACv1_scaffold_157956_ } \\
\text { 2DL:22600-32,475 }\end{array}$ & 9876 & 6 & 5 & 1000 & 5.65 & 108.18 & 9 \\
\hline TaHMA5;2 & & Trae_2AL_D0EABF355 & $\begin{array}{l}\text { TGACv1_scaffold_099043_ } \\
\text { 2AL:1541-7440 }\end{array}$ & 5900 & 6 & 5 & 1011 & 5.66 & 109.11 & 9 \\
\hline TaHMA5;3 & & Trae_2BL_19B3E60AA & $\begin{array}{l}\text { TGACv1_scaffold_130949_ } \\
\text { 2BL:4724-10,512 }\end{array}$ & 5789 & 6 & 5 & 994 & 5.88 & 107.49 & 9 \\
\hline TaHMA6;1 & TaHMA6 & Trae_6BS_9A12C2A1D & $\begin{array}{l}\text { TGACv1_scaffold_513141_ } \\
\text { 6BS:286354-292,595 }\end{array}$ & 6242 & 9 & 8 & 974 & 5.73 & 104.50 & 50 \\
\hline TaHMA6;2 & & Trae_6DS_9FA053DF8 & $\begin{array}{l}\text { TGACv1_scaffold_542481__ } \\
\text { 6DS:33526-39,252 }\end{array}$ & 5727 & 9 & 8 & 1042 & 5.53 & 111.76 & 50 \\
\hline TaHMA6;3 & & Trae_6AS_9321C1C5B & $\begin{array}{l}\text { TGACv1_scaffold_486033_ } \\
6 \text { AS:36396-41,875 }\end{array}$ & 5480 & 9 & 8 & 997 & 5.23 & 106.85 & 53 \\
\hline TaHMA7;1 & TaHMA7 & Trae_7AS_766146E70 & $\begin{array}{l}\text { TGACV1_scaffold_569059_ } \\
\text { 7AS:131450-140,663 }\end{array}$ & 9214 & 17 & 16 & 947 & 7.26 & 99.55 & 22 \\
\hline TaHMA7;2 & & Trae_7BS_8EC4B41E4 & $\begin{array}{l}\text { TGACv1_scaffold_592600_ } \\
\text { 7BS:48974-58,635 }\end{array}$ & 9662 & 17 & 16 & 952 & 7.23 & 100.02 & 26 \\
\hline TaHMA7;3 & & Trae_7DS_04F16455B & $\begin{array}{l}\text { TGACv1_scaffold_622783_ } \\
\text { 7DS:19856-29,376 }\end{array}$ & 9521 & 17 & 16 & 952 & 6.98 & 99.94 & 26 \\
\hline TaHMA8;1 & TaHMA8 & Trae_4BL_89775421A & TGACv1_scaffold_640736_ & 9111 & 17 & 16 & 903 & 6.07 & 94.48 & 37 \\
\hline
\end{tabular}


Table 4 Identified putative wheat HMA genes (Continued)

\begin{tabular}{|c|c|c|c|c|c|c|c|c|c|c|}
\hline HMAs & $\begin{array}{l}\text { Subfamily } \\
\text { gene name }\end{array}$ & $\begin{array}{l}\text { Ensemble Wheat } \\
\text { Gene ID }\end{array}$ & Chromosome location & $\begin{array}{l}\text { Length } \\
\text { (bp) }\end{array}$ & Exons & Introns & $\begin{array}{l}\text { Amino acid } \\
\text { length (aa) }\end{array}$ & $\mathrm{PI}$ & $\begin{array}{l}\mathrm{MW} \\
(\mathrm{kDA})\end{array}$ & $\begin{array}{l}\text { EST } \\
\text { count }\end{array}$ \\
\hline & & & U:185896-195,006 & & & & & & & \\
\hline TaHMA8;2 & & Trae_4AS_622EEFE10 & $\begin{array}{l}\text { TGACv1_scaffold_306691_ } \\
\text { 4AS:30689-37,419 }\end{array}$ & 6731 & 17 & 16 & 900 & 5.85 & 94.16 & 37 \\
\hline TaHMA8;3 & & Trae_4DL_385639507 & $\begin{array}{l}\text { TGACv1_scaffold_344605_ } \\
\text { 4DL:1306-16,767 }\end{array}$ & 15,462 & 20 & 19 & 1702 & 6.93 & 185.21 & 30 \\
\hline TaHMA9;1 & TaHMA9 & Trae_7AL_7A2639A1B & $\begin{array}{l}\text { TGACv1_scaffold_558946_ } \\
\text { 7AL:1236-7780 }\end{array}$ & 6545 & 9 & 8 & 1001 & 5.29 & 106.89 & 17 \\
\hline TaHMA9;2 & & Trae_7DL_DF97DD324 & $\begin{array}{l}\text { TGACv1_scaffold_604439_ } \\
\text { 7DL:28926-35,180 }\end{array}$ & 6255 & 9 & 8 & 1001 & 5.40 & 106.78 & 18 \\
\hline TaHMA9;3 & & Trae_7BL_EFF0E2E31 & $\begin{array}{l}\text { TGACV1_scaffold_579154_ } \\
\text { 7BL:35573-41,675 }\end{array}$ & 6103 & 9 & 8 & 1001 & 5.28 & 106.77 & 16 \\
\hline
\end{tabular}

have similar gene structures. To predict and verify domains in the TaHMAs proteins, we used the Multiple EM for Motif Elicitation (MEME) motif search tool. Ten corresponding consensus motifs were detected (Additional file 2: Figure S8, Table 5). The number of motifs varied among the TaHMA proteins. Motif 2, motif 6, motif 8 , motif 9 and motif 10 were observed in all TaHMA proteins. Motif 1 and motif 3 were observed in all TaHMA proteins except TaHMA1;3.

\section{Tissue-specific expression patterns of TaHMA genes}

Using available RNA-seq data (the wheat expression database, http://wheat.pw.usda.gov/WheatExp/) for five different tissues, the tissue specificity of the TaHMA genes was investigated to focus on the temporal and spatial expression patterns and putative functions of HMA genes in the growth and development. According to FPKM values, we found that the expression levels of the TaHMAs varied significantly in different tissues (Fig. 7). Most HMAs were found to be expressed in five

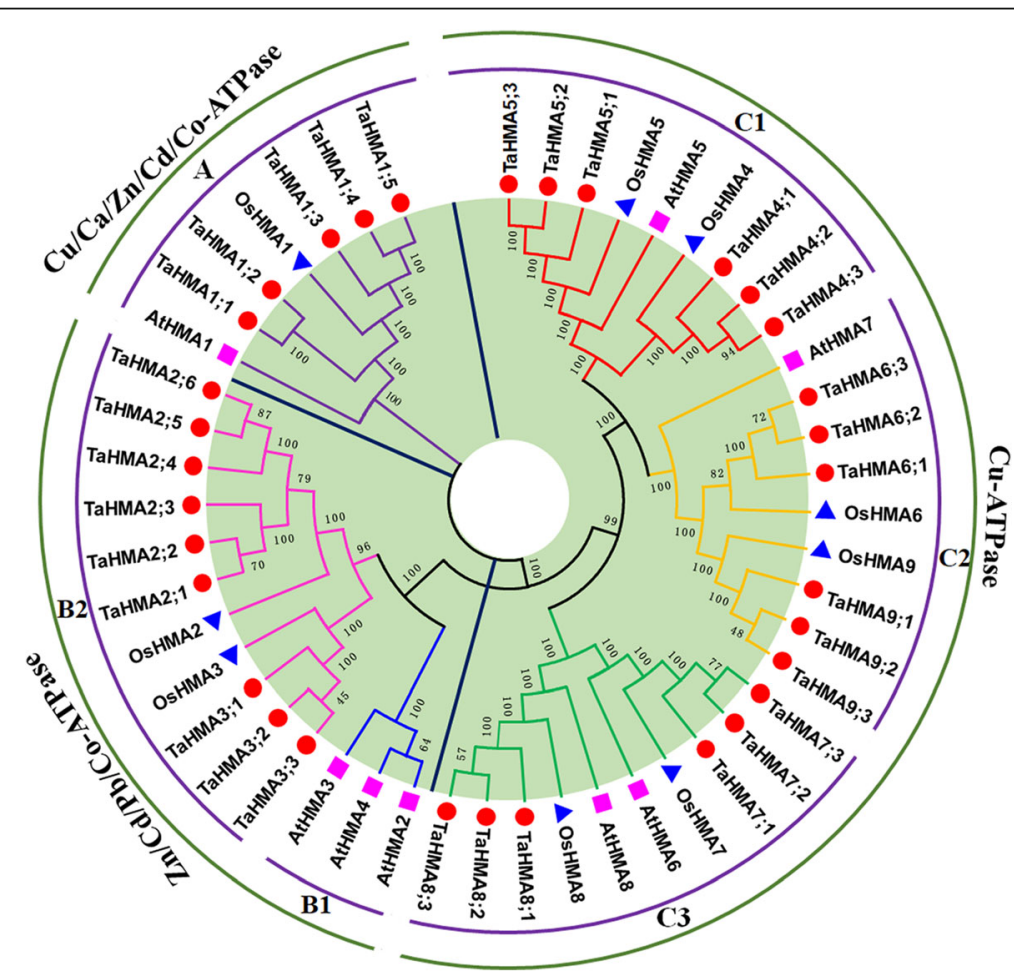

Fig. 6 Neighbour-Joining phylogenetic tree of HMA proteins from wheat (Ta), Aradidopsis (At), and rice (Os). HMA proteins were used to establish the phylogenetic tree with MEGA6.0. TaHMA proteins are marked in red. An unrooted Neighbour-Joining analysis was performed with pairwise deletion and Poisson correction 
Table 5 Motif sequences for HMAs identified by MEME analysis

\begin{tabular}{lll}
\hline Motif & Width & Multilevel consensus sequence \\
\hline 1 & 50 & $\begin{array}{l}\text { LNLBGYJHVRATKVGSNSALAKIVRLVEEAQMSKAPVQRLA } \\
\text { DKVAKYFVP }\end{array}$ \\
2 & 41 & TPTAVMVATGVGARRGVLIKGGDVLESLANIKAIAFDKTGT \\
3 & 50 & $\begin{array}{l}\text { VGDVIKVLPGEKVPVDGWVGRSHVBESMLTGESAPVAKZ } \\
\text { VGSEVIGGT }\end{array}$ \\
4 & 50 & AIGSGTAVAIEAADWLMSNBLEDVPTAIDLSRKTFRTIRQN \\
5 & 41 & KFFEESGMJVFFLLGKYLEVLAKGKASDAMSKLMELAPET \\
6 & 29 & ELQKRGGPVAMVGDGINDAPALAAADVGM \\
7 & 50 & IVGIPVAAGALFPFTGFRPPWLAGACMAFSSVSWCSSLLL \\
8 & 26 & RLYKKPRH \\
9 & 29 & SMGIKSVMLTGDNWVASAESNSEHPLAKAIVEYAQSFS \\
10 & 29 & EEVADFEILPGEGVYAEIDGKKVLVGNKR
\end{tabular}

Motif numbers corresponded to the motifs in Additional file 2: Figure S8 detected organs. TaHMA1;1, TaHMA1;2, TaHMA2;5 and TaHMA4;3 showed weak expression in all tissues, while TaHMA6;1, TaHMA9;1, TaHMA9;2 and TaHMA9; 3 had strong expression. TaHMA1;4, TaHMA2;1, TaHMA2;2, TaHMA2;4, TaHMA2;5, TaHMA7;1, TaHM A7;2, TaHMA7;3, TaHMA8;1, TaHMA8;2 and TaHMA8; 3 were differentially expressed in 5 organs.

\section{Expression pattern of TaHMA genes under cd stress}

To characterize potential roles of TaHMA genes in response to Cd stresses, expression of all TaHMA genes in response to $\mathrm{Cd}$ stress were investigated using our RNA sequencing data. Overall, the 32 wheat HMA genes exhibited diverse expression patterns under Cd challenge. In L17, the expression levels of TaHMA1;2, TaHMA1;4, TaHMA1;5, TaHMA2;2, TaHMA5;1, TaHMA6;1, TaHMA6;3, TaHMA7; 1, TaHMA7;2, TaHMA7;3, TaHMA8;1, TaHMA8;2 and TaHMA9;2 were down-regulated under $\mathrm{Cd}$ stresses, while, the expression levels of TaHMA2;1, TaHMA2;3, TaHMA2; 5, TaHMA2;6, TaHMA4;1, TaHMA4;2, TaHMA4;3, ТаH MA5;2, TaHMA5;3, TaHMA6;2, TaHMA8;3 and TaHMA9;

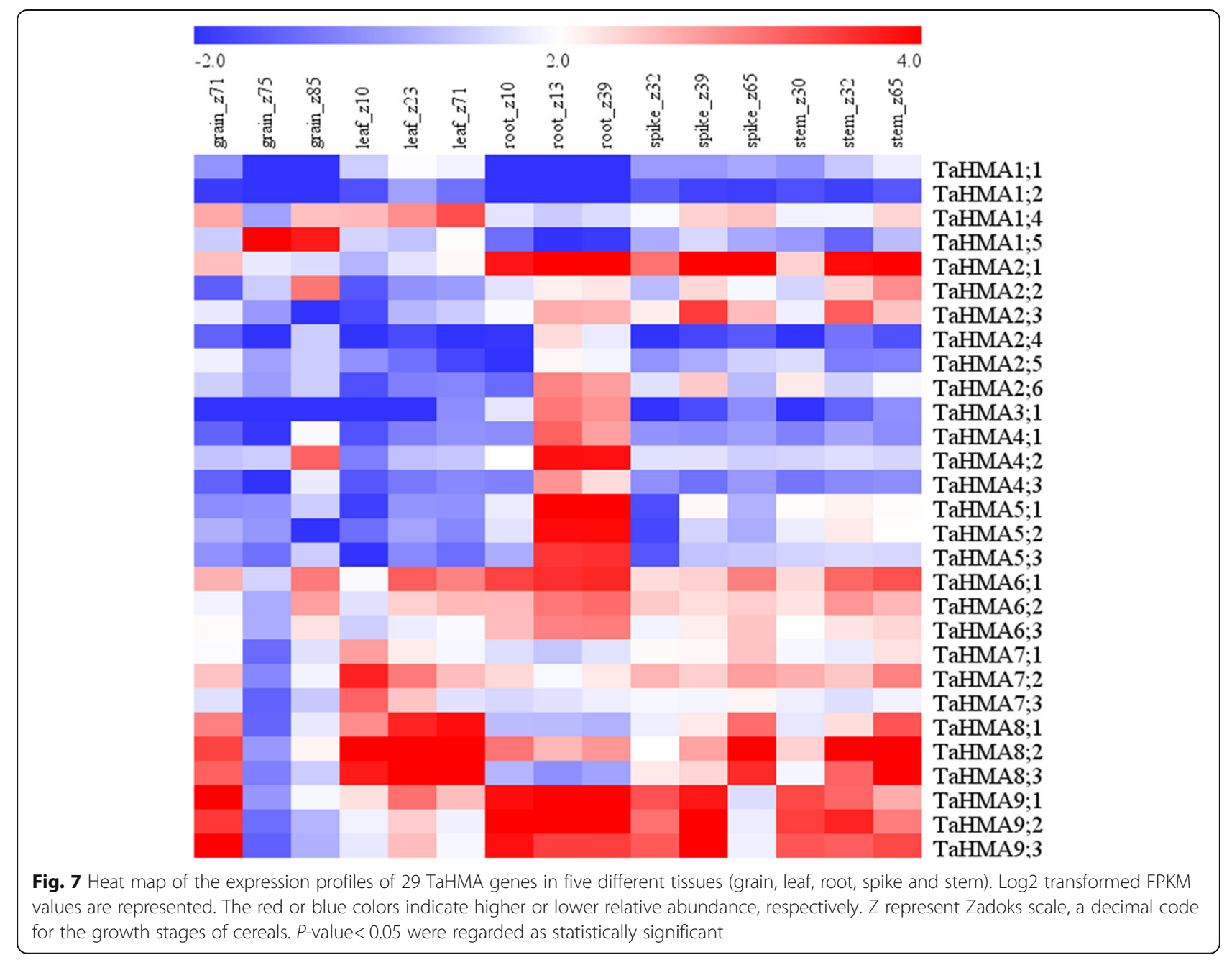


1, TaHMA9;3 were up-regulated (Fig. 8a). In H17, the expression levels of TaHMA1;5, TaHMA5;1, TaHMA6;1, TaHMA6;2, TaHMA6;3, TaHMA7;2, TaHMA7;3, TaHMA9; 1, TaHMA9;2 and TaHMA9;3 were down-regulated under Cd stresses, while, the expression levels of TaHMA1;2, TaHMA1;4, TaHMA2;1, TaHMA2;2, TaHMA2;3, TaHMA2; 5, TaHMA2;6, TaHMA4;1, TaHMA4;2, TaHMA4;3 TaHM A5;2, TaHMA5;3, TaHMA7;1 TaHMA8;1, TaHMA8;2 and TaHMA8;3 were both up-regulated (Fig. 8b).

\section{Integration analysis miRNAs and TaHMAs}

To explore the regulatory association between miRNAs and TaHMAs, microRNA-TaHMAs we performed a expression network analysis using targetfinder in plant [48] which was shown using Cytoscape v3.6 (http://www.cytoscape.org/). Results showed that microRNA-2B_36279* can regulate TaHMA2;1, TaHMA2;2, TaHMA2;4, TaHMA2;5 and TaHMA2;6, while microRNA-4B_11876*, microRNA4B_3407*, microRNA-4B_16562*, microRNA-4B_13629* and microRNA-2B_40139* can regulate TaHMA3;1 (Fig. 9), and microRNA-2B_28883* can regulate TaHMA1; 4. To our knowledge, it is the first time to perform microRNA-TaHMA regulatory network in wheat.

\section{Response of selected TaHMA genes to exogenous cd stress}

HMAs have been implicated in the transmembrane transport of metals, such as $\mathrm{Zn}, \mathrm{Cd}, \mathrm{Pb}$ and $\mathrm{Cu}$ in different plant species $[46,49-51]$. Therefore, we were interested to determine the response of select HMA genes under Cd stress. We thus selected eight TaHMA genes (TaHMA1;4-TaHMA3;1) belonging to the $\mathrm{Cu} / \mathrm{Ca} / \mathrm{Zn} / \mathrm{Cd} / \mathrm{Co}-A T P a s e$ group and the $\mathrm{Zn} / \mathrm{Cd} /$ $\mathrm{Pb} / \mathrm{Co}-\mathrm{ATPase}$ group to determine their relative expression levels in roots under $\mathrm{Cd}$ stress using qRTPCR. Results showed that relative expression levels of TaHMA1;4, TaHMA1;5, TaHMA2;1 and TaHMA3;1 were significantly down-regulated under $\mathrm{Cd}$ treatment compared with those under control conditions in high-accumulating wheat genotype $(\mathrm{H} 17)$, while relative expression levels of TaHMA1;4, TaHMA1;5, TaHMA2;1 andTaHMA2;2 were significantly upregulated under $\mathrm{Cd}$ treatment compared with those under control conditions in low-accumulating wheat genotype (L17) (Fig. 10). Relative expression of TaHMA1;5 in H17, TaHMA2;1 in L17 was consistent with our transcriptome sequencing data. Interestingly, expression data from other TaHMA genes in H17 and L17 treated with $\mathrm{Cd}$ were not consistent with sequencing data. A possible explanation for this inconsistence is the number of samples used on this experiment. Previous work indicated that AtHMA1 is responsible for $\mathrm{Cu}$ to the stroma, exporting $\mathrm{Zn} 2+$ form the chloroplast, or as a $\mathrm{Ca}^{2+} /$ heavy metal transporter to the intracellular organelle [52-54]. AtHMA2 and AtHMA4 in A.thaliana are involved in xylem loading of $\mathrm{Zn}$ as well as $\mathrm{Cd}$ [55-59]. Compared with wild-type plants, plants overexpressing AtHMA3 exhibited a 2-3-fold increase in
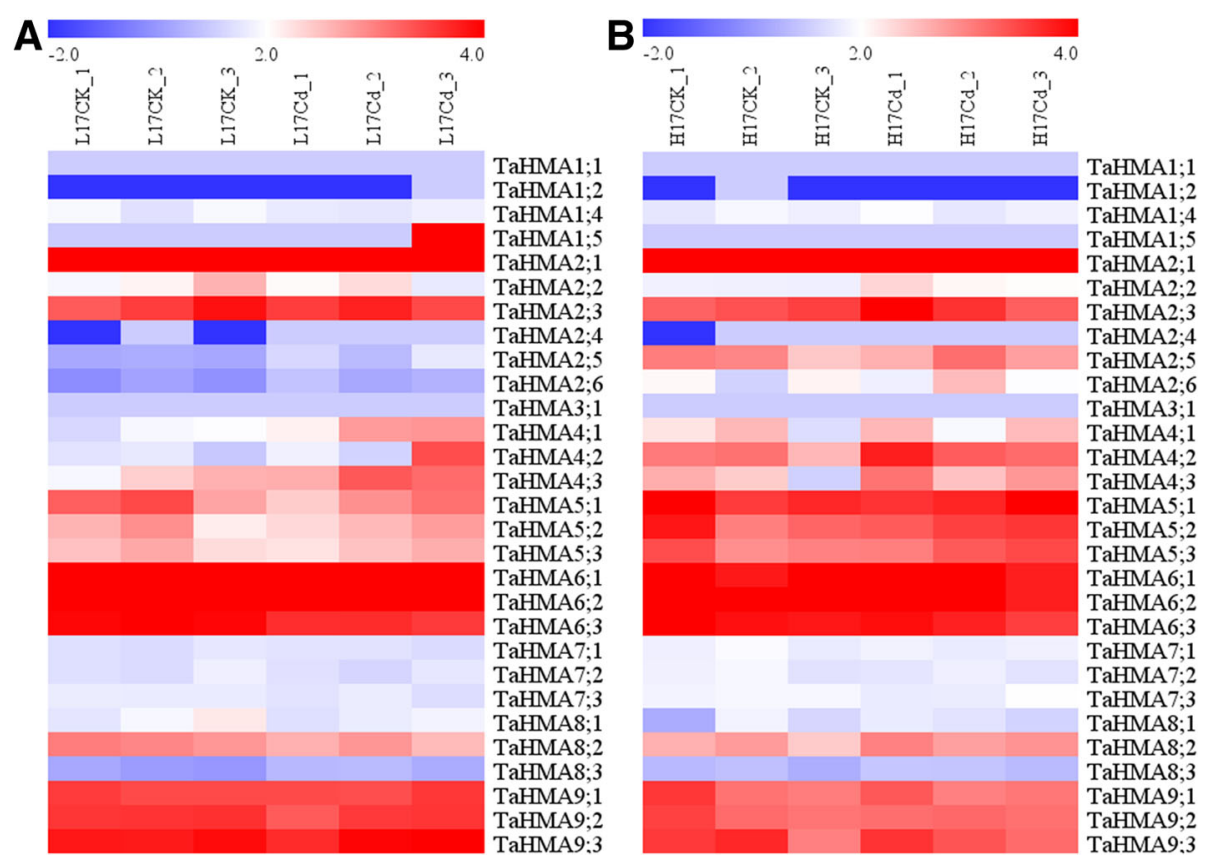

Fig. 8 Heat map of the expression profiles of TaHMA genes under Cd treatment. a Expression profiles of TaHMA genes in L17Cd and L17CK groups. b Expression profiles of TaHMA genes in $\mathrm{H} 17 \mathrm{Cd}$ and H17CK groups. Log2 transformed FPKM values were used to create the heat map. Red or blue indicates higher or lower relative abundance, respectively 


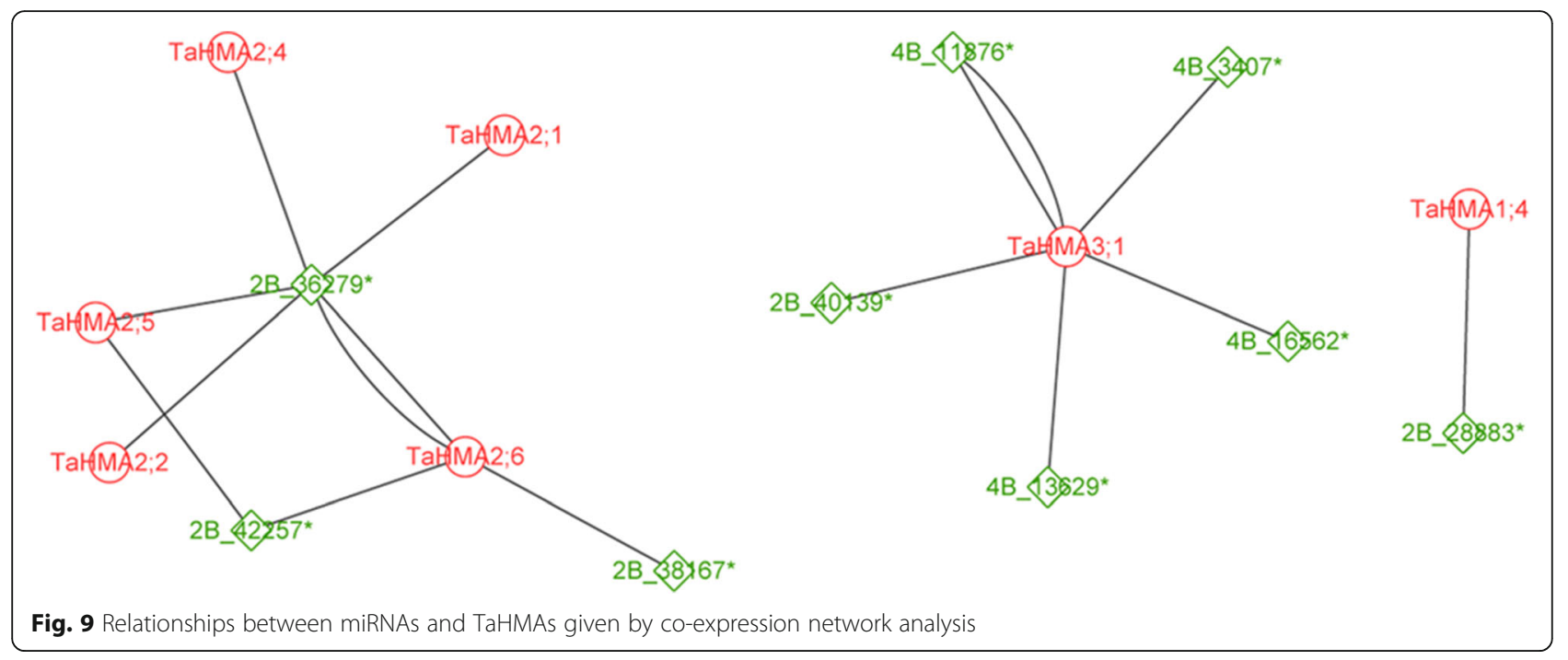

$\mathrm{Cd}$ accumulation [28]. AtHMA5 is responsible for the $\mathrm{Cu}$ translocation from roots to shoots or $\mathrm{Cu}$ detoxification of roots $[60,61]$. AtHMA6 (also known as PAA1) is involved in transporting $\mathrm{Cu}$ over the chloroplast envelope, whereas AtHMA8 (PAA2) most likely transports $\mathrm{Cu}$ into the thylakoid lumen to supply plastocyanin $[62,63]$. AtHMA7 has been proposed to delivery $\mathrm{Cu}$ to ethylene receptors and $\mathrm{Cu}$ homeostasis in the seedlings $[64,65]$. Among rice HMA characterized, OsHMA1 transports $\mathrm{Zn}$ and $\mathrm{Cd}$ [66], OsHMA2 is responsible for $\mathrm{Cd}$ accumulation [67], OsHMA5 is involved in loading $\mathrm{Cu}$ to the xylem of the roots and other organs [68], and OsHMA9 is responsible for transporting $\mathrm{Zn}, \mathrm{Cu}, \mathrm{Pb}$ and Cd [69]. In wheat, TaHMA2 is a plasma membranelocalized $\mathrm{Zn} / \mathrm{Cd}$ transporter that pumps $\mathrm{Zn} / \mathrm{Cd}$ into apoplast [26]. Our results indicated that Cd stress induced the up-regulation of TaHMA2;1 in L17.

\section{Conclusions}

We present here a broad range of genome-wide data surrounding $\mathrm{Cd}$ response in contrasting wheat lines. A total three known and 19 novel differentially expressed microRNAs (DEMs), and 1561 differentially expressed genes (DEGs) were found in L17 after Cd treatment. In H17 we found 12 known and 57 novel DEMs, $297 \mathrm{Cd}$ induced DEGs. Moreover, we present the identification of 32 TaHMA genes in wheat. Finally, we provide microRNA-TaHMA expression networks that suggest that miRNAs can regulate TaHMAs which need to be validated experimentally in future. Taken together, our results indicate that microRNAs may play important roles in wheat under $\mathrm{Cd}$ stress through regulating target genes such as TaHMA2;1. This information will be useful for screening and breeding low-Cd accumulation wheat genotypes.

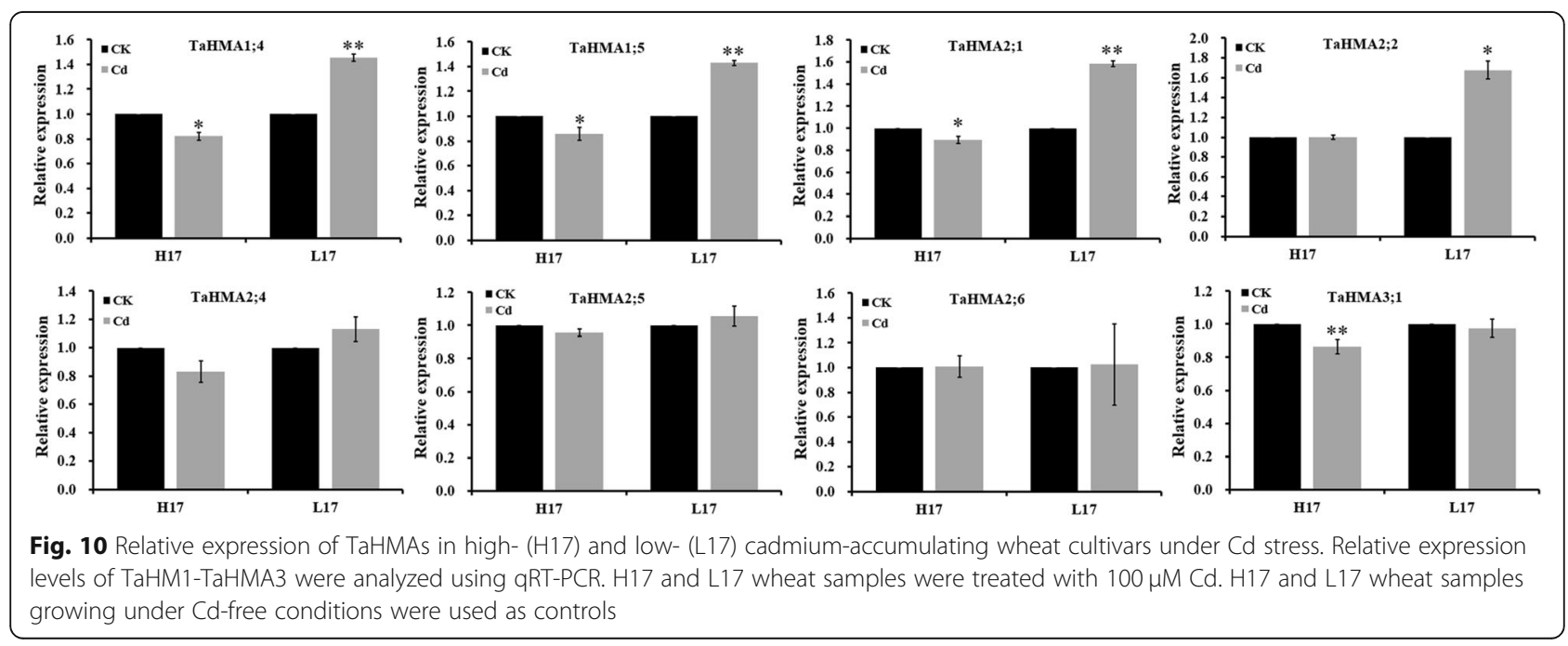




\section{Methods}

\section{Study site and wheat germplasms}

The Shifang heavy industry and Cd-polluted area was selected as our study site in the northeast of Sichuan province. Low grain $\mathrm{Cd}$ accumulative wheat cultivars were selected from the Cd-polluted area as suitable for planting in Cd pollution farmland. A total of 185 wheat germplasms (provided by Chengdu Institute of Biology, Chinese Academy of Sciences) were chosen to include wide genetic diversity. Detailed information of wheat cultivars were shown in Additional file 1: Table S1. The 185 wheat germplasms could be divided into two groups, including 181 bread wheat (Triticum aestivum L.) and 4 durum wheat (Triticum turgidum L. var. durum) groups. The 181 bread wheats were further divided into three groups, including 52 Chuanyu wheat cultivars (Chuanyu group), 56 International Maize and Wheat Improvement Center (CIMMYT) materials (CIMMYT group) and 73 Chinese modern cultivars (CMC group).

\section{Plant growth conditions and cd treatment}

A low-Cd-accumulating cultivar (Chuanyu 17, namely L17) and its sib-line H17 (a high-Cd-accumulating cultivar) from Chuanyu group were used in the study. Seeds were sterilized by soaking in $2 \% \mathrm{H}_{2} \mathrm{O}_{2}$ for $10 \mathrm{~min}$ and fully rinsed with deionized water. After sterilizing, the seeds were soaked in deionized water at room temperature for $6 \mathrm{~h}$, and then planted hydroponically in Hoagland solution in growth chambers at $24 \pm 2{ }^{\circ} \mathrm{C}$ and $50 \%$ relative humidity with a photoperiod of $16 \mathrm{~h}$ light $/ 8$ $\mathrm{h}$ dark. One-week-old seedlings were treated with 0 (CK) and $100 \mu \mathrm{M} \mathrm{CdCl} 2$ for $24 \mathrm{~h}(\mathrm{Cd})$. Roots from the plants of similar size were harvested separately and washed three times with deionized water. Roots from three biological replicates were frozen in liquid nitrogen immediately and stored at $-80{ }^{\circ} \mathrm{C}$ for small RNA and transcriptome sequencing.

\section{Determination of grain and root cd concentrations}

For wheat grain, whole grain was ground into flour $(<1$ $\mathrm{mm})$. Flour was dried at $80^{\circ} \mathrm{C}$ for $12 \mathrm{~h}$ and then digested by $\mathrm{HNO}_{3}$ and $\mathrm{H}_{2} \mathrm{O}_{2}$ in a microwave digester. Roots from three plants as replicates for each of $\mathrm{CK}$ and $\mathrm{Cd}$ treatments were dried at $70{ }^{\circ} \mathrm{C}$ to a constant weight and then also digested by $\mathrm{HNO}_{3}$ and $\mathrm{H}_{2} \mathrm{O}_{2}$ in a microwave digester. All grain samples were processed together with quality controls. Cd concentration was measured using inductively couple plasma-mass spectrometry (ICP-MS, NexIONTM 300, USA) following the manufacturer's instructions [70]. A Certified Reference material (CRM; GBW10020, provided by the National Research Center for CRM, China) was applied to assess the precision of the analytical procedures for plant material.

\section{RNA extraction}

One week-old L17 and H17 seedlings were treated with 0 (CK) and $100 \mu \mathrm{M} \mathrm{CdCl}_{2}$ (Cd) for $24 \mathrm{~h}$. Then, roots of 12 seedlings (each treatment for three biological replicates) were collected for RNA extraction. Total RNA were extracted using the TRIzol Reagent (Invitrogen, USA) following the manufacturer's protocol. RNA integrity was evaluated using the Agilent 2100 Bioanalyzer (Agilent Technologies, Santa Clara, CA, USA), and each sample had an RNA integrity number (RIN) > 8.0.

\section{Small RNA sequencing and analysis}

cDNA libraries were built using SuperScript II Reverse Transcriptase basing on the manufacturer's instructions. Resultant libraries were then sequenced on the Illumina HiSeqTM 2500 sequencing platform (OEbiotech Company, Shanghai, China). Raw reads were converted into sequence data by base calling. After reads with lowquality, 5' primer contaminants and ploy (A), without 3 'adapter and insert tag, shorter than $15 \mathrm{nt}$ and longer than $41 \mathrm{nt}$ from the raw reads were filtered, the clean reads were obtained and mapped against the wheat genome. Clean reads were annotated with miRBase (version 21.0, http://www.mirbase.org/) to identify know miRNAs using Bowtie software. To identify novel miRNAs, the remaining unannotated clean reads that could be aligned to the genome were analyzed by miRDeep 2 and RNAfold. The criteria for designation as a novel miRNA were as follows: length of 18-24 nt, precursors with a signature hairpin structure and the formation of maturation achieved by Dicer, minimum free energy of precursors of less than $18 \mathrm{kcal} / \mathrm{mol}$, a minimum support number for the maturity sequence of precursors of at least 5 , and potential miRNA sequence with less than 3 nt mispairing in the sequence of the mature and perfectly matched middle sequence. Differentially expressed miRNAs (DEMs) were identified with the threshold of $p$ value < 0.05 . While the $\mathrm{p}$ value was calculated with the DEG algorithm in the $\mathrm{R}$ package. The targets of DEMs were predicted by using targetfinder [48].

\section{Transcriptome sequencing}

cDNA libraries were constructed using the TruSeq Stranded mRNA LTSample prep kit (Illumina, Sam Diego, CA, USA) according to the manufacturer's instructions. Resultant libraries were sequenced on the Illumina HiSeqTM 2500 sequencing platform (OEbiotech Company, Shanghai, China). Raw reads were processed using NGS QC toolkit. The reads containing poly-N and the low quality reads were removed to obtain the clean reads. Clean reads were then mapped to wheat reference genome (ftp://ftp.ensemblgenomes.org/pub/plants/release-28/fasta /triticum_aestivum/dna/Triticum_aestivum.IWGSC1.0+p opseq.28.dna.toplevel.fa.gz) [71] using hisat2. Fragments 
per kilobase of transcript sequence per million base pairs sequenced (FPKM) value of each gene was calculated using cufflinks, and the read counts of each gene were obtained by htseq-count. Differentially expressed genes (DEGs) were identified using the DESeq. $\mathrm{R}$ package functions estimateSizeFactors and nbinomTest. $P$ value $<0.05$, fold Change $>2$ and false discovery rate (FDR) $<0.05$ were set as the threshold for significantly differential expression. Hierarchical cluster analysis of DEGs was performed to explore genes expression pattern.

\section{Gene ontology (GO) and KEGG pathway analysis}

For miRNAs, GO enrichment and KEGG pathway enrichment analysis of different expressed miRNA-target-Gene were performed using $\mathrm{R}$ based on the hypergeometric distribution. For mRNAs, GO enrichment and KEGG pathway enrichment analysis of DEGs were performed using $R$ based on the hypergeometric distribution [72].

\section{Identification of HMA gene family in wheat}

The HMA gene family was identified following the method as described by Li et al. with some modifications [73]. First, to construct a local protein database, all the wheat (T.aestivum L.) protein sequences were downloaded from the Ensemble database (http://plants. ensembl.org/index.html). Then, the database were searched with 17 known HMA gene sequences collected from A.thaliana (8) and Osativa (9) using the local BLASTP program with an e-value of le- 5 and identity of $50 \%$ as the threshold. Moreover, a self-blast of these sequences was performed to remove redundant sequences. The physical localizations of all candidate HMA genes were checked and redundant sequences with the same chromosome location were rejected. To analyze whether there were domains belonging to the HMA gene family, the online tool InterProScan 5 (http://www.ebi.ac.uk/ interpro/search/sequence search) [74] was used. Finally, to verify the existence of all the obtained sequences, BLASTN similarity search against the wheat ESTs deposited in the NCBI database were performed. The theorectical pI (isoelectric point) and $\mathrm{Mw}$ (molecular weight) of the putative HMA from T.aestivum $\mathrm{L}$ were calculated using compute $\mathrm{pI} / \mathrm{Mw}$ tool online (http://web.expasy. org/compute_pi/), respectively.

\section{Gene structure construction, protein domain and motif analysis}

Gene structure information was obtained from the Ensemble plants database (http://plants.ensembl.org/index. html). All full-length amino acid sequences of the TaHMAs were also used to identify conserved domain motifs by the Multiple Em for Motif Elicitation (MEME) tool [75]. The parameters were set as follows: maximum numbers of different motifs, 10; minimum motif width, 6; maximum motif width, 50 .

\section{Phylogenetic analysis}

The HMA protein sequences from A.thaliana, Osativa and T.aestivum $L$. were performed for multiple alignments by CLUSTALW and the results of alignment were used to construct phylogenetic tree using the $\mathrm{NJ}$ method in MEGA (version 6.0) [76]. Bootstrap test method was emplyed and the replicate was set to 1000 .

The TaHMA gene expression analysis by RNA-seq data To study the expression of TaHMA genes in different organs, the wheat expression database (http://wheat.pw. usda.gov/WheatExp/) was used to investigate the differential expression of TaHMAs. The FPKM (fragments per kilobase of transcript per million fragments mapped) value was calculated for each HMA gene, the log2 transformed FPKM value of the TaHMA genes were used for heat map generation. And $p$-value $<0.05$ were taken as statistically significant threshold [77].

\section{Quantitative real time polymerase chain reaction (qRT-} PCR) analysis

Eight miRNAs and six genes with different expression patterns and eight TaHMA genes were selected for validation by quantitative real-time RT-PCR (qRT-PCR). Total RNA was extracted from roots of L17 and H17 seedlings treated with 0 (CK) or $100 \mu \mathrm{M} \mathrm{CdCl} 2(\mathrm{Cd})$ for $24 \mathrm{~h}$. For mRNA, first strand cDNA was synthesized using HiScript II Q RT SuperMix (Vazyme, R223-1). For miRNA, the first strand cDNA was synthesized using miScript II Reverse Transcription Kit (Qiagen, 218,161). The qRT-PCR was carried out using QuantiFast ${ }^{\circ}$ SYBR $^{\circ}$ Green PCR kit (Qiagen, 204,054) according to the manufacturer's instructions. The primers used in the qRT-PCR analyses were listed in (Additional file 4). GAPDH and $5 \mathrm{~S}$ were used as internal genes for mRNAs and miRNAs, respectively. Three technical replicates were performed for each gene. The expression levels were calculated from the $2^{-\Delta \Delta C t}$ value.

\section{Statistical analysis}

All data were represented as mean \pm SD and analyzed with SPSS20.0 software. For multiple group comparison, ANOVA with least significant difference was applied. $P<0.05$ was considered statistically significant.

\section{Additional files}

Additional file 1: Grain Cd concentration among wheat germplasms. (XLSX $16 \mathrm{~kb}$ )

Additional file 2: Figure S1. Grain $\mathrm{Cd}$ concentration between bread wheat and durum wheat. Figure S2. A Box plot of miRNA. B Correlation 
analysis of expression levels of miRNAs between samples and samples. Figure S3. A Box plot of mRNA. B Correlation analysis of expression levels of mRNAs between samples and samples. Figure S4. A The volcano plot illustrates the distribution of mRNA expression fold changes vs P values between L17Cd and L17CK. B The volcano plot illustrates the distribution of the data in mRNA profiles between $\mathrm{H} 17 \mathrm{Cd}$ and HL17CK. Red, green and black points in the volcano plot represents significantly upregulated mRNAs, significantly downregulated mRNAs and not differential expressed mRNAs, respectively. Figure S5. GO enrichment analysis of differentially-expressed miRNA-targeted genes. The top $10 \mathrm{GOs}$ enriched in targeted genes are given in response to miRNAs: (A) downregulated and (B) up-regulated between $\mathrm{L} 17 \mathrm{Cd}$ and $\mathrm{L} 17 \mathrm{CK}$; (C) downregulated (D) up-regulated between $\mathrm{H} 17 \mathrm{Cd}$ and H17CK. The $\mathrm{y}$-axis represent the number of genes enriched. Figure S6. Venn diagrams of mRNA Gene Ontology (GO) enrichment results. Overlap of GO results of differentially expressed microRNAs (DEMs) between L17Cd and L17CK, $\mathrm{H} 17 \mathrm{Cd}$ and H17CK. B Venn diagram for GO of DEGs between L17Cd and L17CK, H17Cd and H17CK. Figure S7. GO analysis for differentiallyexpressed mRNAs. The top $10 \mathrm{GOs}$ enriched in differentially expressed mRNAs are given for the contrasts: (A) up-regulated and (B) downregulated between $\mathrm{L} 17 \mathrm{Cd}$ and $\mathrm{L} 17 \mathrm{CK} ;(\mathrm{C})$ up-regulated and (D) downregulated between $\mathrm{H} 17 \mathrm{Cd}$ and $\mathrm{H} 17 \mathrm{CK}$. The $\mathrm{y}$-axis represent the gene number enriched in GOs. Figure S8. Ten wheat HMAs motifs were identified by MEME tools and indicated by different color. Motif location and combined p-value were represented. (ZIP $1392 \mathrm{~kb}$ )

Additional file 3: Functional annotation of transport genes. (XLSX $27 \mathrm{~kb})$ Additional file 4: Primers for genes. (XLSX $10 \mathrm{~kb}$ )

\section{Abbreviations}

Cd: Cadmium; Chuanyu: Chuanyu wheat cultivars; CIMMYT: International Maize and Wheat Improvement Center: CMC: Chinese modern cultivars: DEGs: Differentially expressed genes; DEMs: Differentially expressed microRNAs; FDR: False discovery rate; FPKM: Fragments per kilobase of transcript per million fragments mapped; GO: Gene ontology; HMAs: Heavy metal ATPases; MEME: Multiple Em for Motif Elicitation; qRT-PCR: Quantitative real time quantitative-polymerase chain reaction

\section{Acknowledgements}

We thank Xian Fu and Pengfei Xiang for technical support.

\section{Authors' contributions}

$\mathrm{MZ}$ and $\mathrm{YW}$ contributed to the experimental design and manuscript drafting $M Z, S G Z$ and RL performed the research. MZ performed bioinformatic analysis. MZ, LL and CHZ contributed reagents/materials/analysis tools. LY supported the revision of the manuscript and drafted the manuscript revision with $\mathrm{MZ}$ and $\mathrm{YW}$. All authors read and approved the final manuscript.

\section{Funding}

This work was supported by the "13th Five-year Plan" for National Key Research and Development (Grant No. 2016YFD0102000). This work was supported by the European Research Council (ERC) under the European Union's Horizon 2020 research and innovation programme [grant number ERC-StG 679056 HOTSPOT], via a grant to L.Y. The funding body had no role in the design of the study, collection, analysis, or interpretation of data, or in writing the manuscript.

\section{Availability of data and materials}

The dataset and materials presented in the investigation is available by request from the corresponding author.

\section{Ethics approval and consent to participate}

Not applicable.

\section{Consent for publication}

Not applicable.

\section{Competing interests}

The authors declare that they have no competing interests.

\section{Author details}

'Chengdu Institute of Biology, Chinese Academy of Sciences, Chengdu 610041, Sichuan, China. ${ }^{2}$ University of Chinese Academy of Sciences, Beijing 100049, China. ${ }^{3}$ School of Life Sciences, University of Nottingham, Nottingham NG7 2RD, UK.

Received: 20 November 2018 Accepted: 26 June 2019

Published online: 29 July 2019

\section{References}

1. Khan MA, Khan S, Khan A, Alam M. Soil contamination with cadmium consequences and remediation using organic amendments. Sci Total Environ. 2017;601-602:1591-605.

2. Kazantzis G. Cadmium, osteoporosis and calcium metabolism. Biometals. 2004:17:493-8.

3. Straif $K$, Benbrahim-Tallaa L, Baan R, Grosse $Y$, Secretan B, El GF, et al. A review of human carcinogens--Part $C$ : metals, arsenic, dusts, and fibres. Lancet Oncol. 2009;10:453.

4. Das P, Samantaray S, Rout GR. Studies on cadmium toxicity in plants: a review. Environ Pollut. 1997:98:29-36.

5. Zorrig W, Rouached A, Shahzad Z, Abdelly C, Davidian JC, Berthomieu P. Identification of three relationships linking cadmium accumulation to cadmium tolerance and zinc and citrate accumulation in lettuce. J Plant Physiol. 2010;167:1239-47.

6. Uraguchi S, Kamiya T, Sakamoto T, Kasai K, Sato Y, Nagamura Y, et al. Lowaffinity cation transporter (OsLCT1) regulates cadmium transport into rice grains. Proc Natl Acad Sci U S A. 2011;108:20959-64.

7. Huang M, Zhou S, Sun B, Zhao Q. Heavy metals in wheat grain: Assessment of potential health risk for inhabitants in Kunshan, China. Sci Total Environ. 2008:405:54.

8. Dai XP, Feng L, Ma XW, Zhang YM. Concentration Level of Heavy Metals in Wheat Grains and the Health Risk Assessment to Local Inhabitants from Baiyin, Gansu, China. Adv Mater Res. 2012;518-523:951-6.

9. Satarug S, Baker JR, Urbenjapol S, Haswellelkins M, Reilly PE, Williams DJ, Moore MR. A global perspective on cadmium pollution and toxicity in nonoccupationally exposed population. Toxicol Lett. 2003;137:65-83.

10. Gill BS, Appels R, Botha-Oberholster AM, Buell CR, Bennetzen JL, Chalhoub $B$, et al. A workshop report on wheat genome sequencing: International Genome Research on Wheat Consortium. Genetics. 2004;168:1087-96.

11. Mayer KFX, Rogers J, Doležel J, Pozniak C, Eversole K, Feuillet C, et al. A chromsome-based draft sequence of the hexaploid bread wheat (Triticum aestivum) genome. Science. 2014;345:1251788.

12. Jafarnejadi AR, Homaee M, Sayyad G, Bybordi M. Large scale spatial variability of accumulated cadmium in the wheat farm grains. Soil Sediment Contam Int J. 2011;20:98-113.

13. Greger M, Lofstedt M. Comparison of uptake and distribution of cadmium in different cultivars of bread and durum wheat. Crop Sci. 2004:44:111-3.

14. Bartel DP. MicroRNAs: genomics, biogenesis, mechanism, and function. Cell. 2004;116:281-97.

15. Voinnet O. Origin, biogenesis, and activity of plant microRNAs. Cell. 2009; 136:669-87.

16. Zhang B, Pan X, Cobb GP, Anderson TA. Plant microRNA: A small regulatory molecule with big impact. Dev Biol. 2006;289:3-16.

17. Sunkar R, Chinnusamy V, Zhu J, Zhu JK. Small RNAs as big players in plant abiotic stress responses and nutrient deprivation. Trends Plant Sci. 2007;12: $301-9$.

18. Sunkar R, Kapoor A, Zhu JK. Posttranscriptional induction of two Cu/Zn superoxide dismutase genes in Arabidopsis is mediated by downregulation of miR398 and important for oxidative stress tolerance. Plant Cell. 2006;18: 2051-65.

19. Abdel-Ghany SE, Pilon M. MicroRNA-mediated systemic down-regulation of copper protein expression in response to low copper availability in Arabidopsis. J Biol Chem. 2008;283:15932-45.

20. Fujii H, Chiou TJ, Lin SI, Aung K, Zhu JK. A miRNA involved in phosphatestarvation response in Arabidopsis. Curr Biol. 2005:15:2038.

21. Liu HH, Tian X, Li YJ, Wu CA, Zheng CC. Microarray-based analysis of stressregulated microRNAs in Arabidopsis thaliana. RNA. 2008;14:836-43.

22. Meng F, Liu H, Wang K, Liu L, Wang S, Zhao Y, Yin J, Li Y. Developmentassociated microRNAs in grains of wheat (Triticum aestivum L.). BMC Plant Biol. 2013;13:140. 
23. Zhou J, Cheng Y, Yin M, Yang E, Gong W, Liu C, et al. Identification of novel miRNAs and miRNA expression profiling in wheat hybrid necrosis. PLoS One. 2015;10:e0117507.

24. Akdogan G, Tufekci ED, Uranbey S, Unver T. miRNA-based drought regulation in wheat. Funct Integr Genomics. 2016;16:221-33.

25. Møller JV, Juul B, le Maire M. Structural organization, ion transport, and energy transduction of P-type ATPases. Biochim Biophys Acta. 1996;1286:1-51.

26. Tan J, Wang J, Chai T, Zhang Y, Feng S, Li Y, Zhao H, Liu H, Chai X. Functional analyses of TaHMA2, a P(1B)-type ATPase in wheat. Plant Biotechnol J. 2013;11:420-31.

27. Miyadate H, Adachi S, Hiraizumi A, Tezuka K, Nakazawa N, Kawamoto T, et al. OsHMA3, a P1B-type of ATPase affects root-to-shoot cadmium translocation in rice by mediating efflux into vacuoles. New Phytol. 2011; 189:190-9.

28. Morel M, Crouzet J, Gravot A, Auroy P, Leonhardt N, Vavasseur A, Richaud P. AtHMA3, a P1B-ATPase allowing $\mathrm{Cd} / \mathrm{Zn} / \mathrm{Co} / \mathrm{Pb}$ vacuolar storage in Arabidopsis. Plant Physiol. 2009;149:894-904.

29. Ueno D, Milner MJ, Yamaji N, Yokosho K, Koyama E, Clemencia Zambrano $\mathrm{M}$, et al. Elevated expression of TcHMA3 plays a key role in the extreme $\mathrm{Cd}$ tolerance in a $\mathrm{Cd}$-hyperaccumulating ecotype of Thlaspi caerulescens. Plant J. 2011;66:852-62.

30. Hart JJ, Welch RM, Norvell WA, Sullivan LA, Kochian LV. Characterization of cadmium binding, uptake, and translocation in intact seedlings of bread and durum wheat cultivars. Plant Physiol. 1998;116:1413-20.

31. Hart JJ, Welch RM, Norvell WA, Kochian LV. Transport interactions between cadmium and zinc in roots of bread and durum wheat seedlings. Physiol Plant. 2002;116:73-8.

32. Greger M, Lofstedt M. Comparison of uptake and distribution of cadmium in different cultivars of bread and durum wheat. Crop Sci. 2004:44:501-7.

33. Erdman JA, Moul RC. Mineral composition of small-grain cultivars from a uniform test plot in South Dakota. J Agric Food Chem. 1982;30:169-74.

34. Han R, Jian C, LV J, Yan Y, Chi Q, Li Z, et al. Identification and characterization of microRNAs in the flag leaf and developing seed of wheat (Triticum aestivum L.). BMC Genomics. 2014;15:289.

35. Feng $H$, Zhang Q, Li H, Wang X, Wang X, Duan X, Wang B, Kang Z. vsiRNAs derived from the miRNA-generating sites of pri-tae-miR159a based on the BSMV system play positive roles in the wheat response to Puccinia striiformis $\mathrm{f}$. sp. tritici through the regulation of taMyb3 expression. Plant Physiol Biochem. 2013;68:90-5.

36. Wei B, Cai T, Zhang R, Li A, Huo N, Li S, et al. Novel microRNAs uncovered by deep sequencing of small RNA transcriptomes in bread wheat (Triticum aestivum L.) and Brachypodium distachyon (L.) Beauv. Funct Integr Genomics. 2009:9:499-511.

37. Jia X, Wang WX, Ren L, Chen QJ, Mendu V, Willcut B, Dinkins R, Tang X, Tang G. Differential and dynamic regulation of miR398 in response to ABA and salt stress in Populus tremula and Arabidopsis thaliana. Plant Mol Biol. 2009;71:51-9.

38. Guan Q, Lu X, Zeng H, Zhang Y, Zhu J. Heat stress induction of miR398 triggers a regulatory loop that is critical for thermotolerance in Arabidopsis. Plant J. 2013;74:840-51.

39. Naya L, Paul S, Valdes-Lopez O, Mendoza-Soto AB, Nova-Franco B, SosaValencia G, Reyes JL, Hernandez G. Regulation of copper homeostasis and biotic interactions by microRNA 398b in common bean. PLoS One. 2014;9: e84416

40. Khraiwesh B, Zhu JK, Zhu J. Role of miRNAs and siRNAs in biotic and abiotic stress responses of plants. Biochim Biophys Acta. 2012;1819:137.

41. Feng J, Jia W, Lv S, Bao H, Miao F, Zhang X, et al. Comparative transcriptome combined with morpho-physiological analyses revealed key factors for differential cadmium accumulation in two contrasting sweet sorghum genotypes. Plant Biotechnol J. 2018;16:558-71.

42. Lux A, Martinka M, Vaculik M, White PJ. Root responses to cadmium in the rhizosphere: a review. J Exp Bot. 2011;62:21-37.

43. Verrier PJ, Bird D, Burla B, Dassa E, Forestier C, Geisler M, et al. Plant ABC proteins--a unified nomenclature and updated inventory. Trends Plant Sci. 2008;13:151-9

44. Thomine S, Wang R, Ward JM, Crawford NM, Schroeder Jl. Cadmium and iron transport by members of a plant metal transporter family in Arabidopsis with homology to Nramp genes. Proc Natl Acad Sci U S A. 2000;97:4991-6.

45. Raghuram B, Sheikh AH, Sinha AK. Regulation of MAP kinase signaling cascade by microRNAs in Oryza sativa. Plant Signal Behav. 2014;9:e972130.
46. Williams LE, Mills RF. P1B-ATPases-an ancient family of transition metal pumps with diverse functions in plants. Trends Plant Sci. 2005;10:491-502.

47. Hermand V, Julio E, de Borne FD, Punshon T, Ricachenevsky FK, Bellec A, Gosti F, Berthomieu P. Inactivation of two newly identified tobacco heavy metal ATPases leads to reduced $\mathrm{Zn}$ and $\mathrm{Cd}$ accumulation in shoots and reduced pollen germination. Metallomics. 2014;6:1427-40.

48. Fahlgren N, Carrington JC. miRNA Target Prediction in Plants. Methods Mol Biol. 2010;592:51-7.

49. Grotz N, Guerinot ML. Molecular aspects of $\mathrm{Cu}$, Fe and $\mathrm{Zn}$ homeostasis in plants. Biochimica et Biophysica Acta (BBA)-Molecular. Cell Res. 2006;1763: 595-608.

50. Argüello JM, Eren E, González-Guerrero M. The structure and function of heavy metal transport P 1B-ATPases. Biometals. 2007;20:233.

51. Burkhead JL, Reynolds KAG, Abdel-Ghany SE, Cohu CM, Pilon M. Copper homeostasis. New Phytol. 2009:182:799-816.

52. Seigneurin-Berny D, Gravot A, Auroy P, Mazard C, Kraut A, Finazzi G, et al. HMA1, a new Cu-atpase of the chloro plast envelope, is essential for growth under adverse light conditions. J Biol Chem. 2006;281:2882-92.

53. Moreno I, Norambuena L, Maturana D, Toro M, Vergara C, Orellana A, ZuritaSilva A, Ordenes VR. AtHMA1 is a thapsigargin-sensitive Ca2+/heavy metal pump. J Biol Chem. 2008:283:9633-41.

54. Kim YY, Choi H, Segami S, Cho HT, Martinoia E, Maeshima M, Lee Y. AtHMA1 contributes to the detoxification of excess $\mathrm{Zn}$ (II) in Arabidopsis. Plant J. 2009:58:737-53.

55. Hussain D, Haydon MJ, Wang Y, Wong E, Sherson SM, Young J, Camakaris J, Harper JF, Cobbett CS. P-type ATPase heavy metal transporters with roles in essential zinc homeostasis in Arabidopsis. Plant Cell. 2004;16:1327-39.

56. Verret F, Gravot A, Auroy P, Leonhardt N, David P, Nussaume L, Vavasseur A, Richaud P. Overexpression of AtHMA4 enhances root-to-shoot translocation of zinc and cadmium and plant metal tolerance. FEBS Lett. 2004:576:306-12.

57. Wong CKE, Cobbett CS. HMA P-type ATPases are the major mechanism for rootto-shoot Cd translocation in Arabidopsis thaliana. New Phytol. 2009:181:71-8.

58. Wong CKE, Jarvis RS, Sherson SM, Cobbett CS. Functional analysis of the heavy metal binding domains of the $\mathrm{Zn} / \mathrm{Cd}$-transporting ATPase, HMA2, in Arabidopsis thaliana. New Phytol. 2009;181:79-88.

59. Yamaji N, Xia XJ, Mitani-Ueno N, Yokosho K, Ma JF. Preferential delivery of $\mathrm{Zn}$ to developing tissues in rice is mediated by a P-type ATPases, OsHMA2. Plant Physiol. 2013:113.216564.

60. Andrés-Colás N, Sancenón V, Rodríguez-Navarro S, Mayo S, Thiele DJ, Ecker JR, Puig S, Peñarrubia L. The Arabidopsis heavy metal P-type ATPase HMA5 interacts with metallochaperones and functions in copper detoxification of roots. Plant J. 2006:45:225-36.

61. Kobayashi Y, Kuroda K, Kimura K, Southron-Francis JL, Furuzawa A, Kimura K, et al. Amino acid polymorphisms in strictly conserved domains of a P-type ATPase HMA5 are involved in the mechanism of copper tolerance variation in Arabidopsis. Plant Physiol. 2008;148:969-80.

62. Shikanai T, Müller-Moulé P, Munekage Y, Niyogi KK, Pilon M. PAA1, a P-type ATPase of Arabidopsis, functions in copper transport in chloroplasts. Plant Cell. 2003;15:1333-46.

63. Abdel-Ghany SE, Müller-Moulé P, Niyogi KK, Pilon M, Shikanai T. Two P-type ATPases are required for copper delivery in Arabidopsis thaliana chloroplasts. Plant Cell. 2005;17:1233-51.

64. Woeste KE, Kieber JJ. A strong loss-of-function mutation in RAN1 results in constitutive activation of the ethylene response pathway as well as a rosette-lethal phenotype. Plant Cell. 2000;12:443-55.

65. Binder BM, Rodríguez Fl, Bleecker AB. The copper transporter RESPONSIVE-TOANTAGONIST1 (RAN1) is essential for the biogenesis of ethylene receptors in Arabidopsis. J Biol Chem. 2010. https://doi.org/10.1074/jbc.M110.170027.

66. Takahashi R, Ishimaru Y, Shimo H, Ogo Y, Senoura T, Nishizawa NK, Nakanishi $\mathrm{H}$. The OsHMA2 transporter is involved in root-to-shoot translocation of $\mathrm{Zn}$ and $\mathrm{Cd}$ in rice. Plant Cell Environ. 2012;35:1948-57.

67. Ueno D, Yamaji N, Kono I, Huang CF, Ando T, Yano M, Ma JF. Gene limiting cadmium accumulation in rice. Proc Natl Acad Sci U S A. 2010;107:16500-5.

68. Deng F, Yamaji N, Xia J, Ma JF. A member of heavy metal P-type ATPase OsHMA5 is involved in xylem loading of copper in rice. Plant Physiol. 2013: 113.226225

69. Lee S, Kim Y-Y, Lee Y, An G. Rice P1B-type heavy-metal ATPase, OsHMA9, is a metal efflux protein. Plant Physiol. 2007;145:831-42.

70. Arnold BJ, Lahner B, DaCosta JM, Weisman CM, Hollister JD, Salt DE, Bomblies K, Yant L. Borrowed alleles and convergence in serpentine adaptation. Proc Natl Acad Sci U S A. 2016;113:8320-5. 
71. Feng N, Song G, Guan J, Chen K, Jia M, Huang D, et al. Transcriptome profiling of wheat inflorescence development from spikelet initiation to floral patterning identified stage-specific regulatory genes. Plant Physiol. 2017:174:1779-94

72. Kanehisa M, Araki M, Goto S, Hattori M, Hirakawa M, Itoh M, et al. KEGG for linking genomes to life and the environment. Nucleic Acids Res. 2008;36:D480-4

73. Li N, Xiao H, Sun J, Wang S, Wang J, Chang P, et al. Genome-wide analysis and expression profiling of the HMA gene family in Brassica napus under $\mathrm{cd}$ stress. Plant Soil. 2018:426:365-81.

74. Jones P, Binns D, Chang H-Y, Fraser M, Li W, McAnulla C, et al. InterProScan 5: genome-scale protein function classification. Bioinformatics. 2014;30:1236-40.

75. Bailey TL, Williams N, Misleh C, Li WW. MEME: discovering and analyzing DNA and protein sequence motifs. Nucleic Acids Res. 2006;34:W369-W73.

76. Tamura K, Stecher G, Peterson D, Filipski A, Kumar S. MEGA6: molecular evolutionary genetics analysis version 6.0. Mol Biol Evol. 2013;30:2725-9.

77. Pearce S, Vazquez-Gross H, Herin SY, Hane D, Wang Y, Gu YQ, Dubcovsky J. WheatExp: an RNA-seq expression database for polyploid wheat. BMC Plant Biol. 2015;15:299.

\section{Publisher's Note}

Springer Nature remains neutral with regard to jurisdictional claims in published maps and institutional affiliations.

Ready to submit your research? Choose BMC and benefit from:

- fast, convenient online submission

- thorough peer review by experienced researchers in your field

- rapid publication on acceptance

- support for research data, including large and complex data types

- gold Open Access which fosters wider collaboration and increased citations

- maximum visibility for your research: over $100 \mathrm{M}$ website views per year

At BMC, research is always in progress.

Learn more biomedcentral.com/submissions 